In cooperation with the U.S. Department of Agriculture and Texas State Soil and Water Conservation Board

\title{
Summary Statistics and Graphical Comparisons of Historical Hydrologic and Water-Quality Data, Seco Creek Watershed, South-Central Texas
}

Open-File Report 98-627

U.S. Department of the Interior U.S. Geological Survey 
Cover photographs:

Top Left-An area near the headwaters of the Seco Creek Basin, Seco Creek watershed, Uvalde County, Texas. Photograph by David S. Brown, 1995.

Top Right-Jon R. Gilhousen and Charles A. Hartmann, Jr., making a discharge measurement and collecting a water-quality sample, respectively, at Seco Creek Reservoir Inflow near Utopia, Texas. Photograph by Gregory M. Nalley, U.S. Geological Survey, 1992.

Bottom Left-Parkers Creek Reservoir Inflow near D'Hanis, Texas, after the flood in June 1997. Photograph by David S. Brown.

Bottom Right-Tom Fillinger, U.S. Department of Agriculture, Natural Resources Conservation Service, supervising maintenance of the Arnold rain gage near D'Hanis, Texas. Photograph by Robert Morris, 1996. 
U.S. Department of the Interior

U.S. Geological Survey

\section{Summary Statistics and Graphical Comparisons of Historical Hydrologic and Water-Quality Data, Seco Creek Watershed, South-Central Texas}

By David S. Brown, Richard N. Slattery, and Jon R. Gilhousen

U.S. GEOLOGICAL SURVEY

Open-File Report 98-627

Prepared in cooperation with the U.S. Department of Agriculture and Texas State Soil and Water Conservation Board 


\section{U.S. DEPARTMENT OF THE INTERIOR}

Bruce Babbitt, Secretary

\section{U.S. GEOLOGICAL SURVEY}

Charles G. Groat, Director

Any use of trade, product, or firm names is for descriptive purposes only and does not imply endorsement by the U.S. Government.

For additional information write to

\section{District Chief}

U.S. Geological Survey 8027 Exchange Dr.

Austin, TX 78754-4733

E-mail: dc_tx@usgs.gov

Copies of this report can be purchased from

U.S. Geological Survey

Branch of Information Services

Box 25286

Denver, CO 80225-0286

E-mail: infoservices@usgs.gov 


\section{CONTENTS}

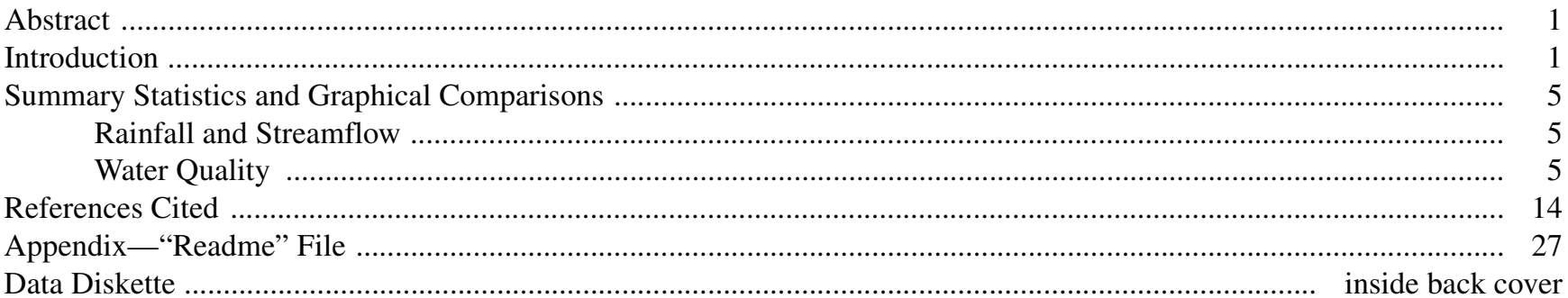

\section{FIGURES}

1-2. Maps showing:

1. Location of the Seco Creek watershed, south-central Texas .........................................................

2. Location of data-collection sites in the Seco Creek watershed, south-central Texas ..................................

3-4. Hydrographs showing:

3. Daily rainfall at selected stations, Seco Creek watershed, south-central Texas, during-and-after implementation period of best-management practices

4. Daily mean streamflow at selected stations, Seco Creek watershed, south-central Texas, duringand-after implementation period of best-management practices

5-9. Scatterplots showing:

5. Specific conductance and $\mathrm{pH}$ data aggregated by stream-stage condition at selected stations, Seco Creek watershed, south-central Texas, during-and-after implementation period of bestmanagement practices

6. Dissolved oxygen and biochemical oxygen demand data aggregated by stream-stage condition at selected stations, Seco Creek watershed, south-central Texas, during-and-after implementation period of best-management practices

7. Fecal coliform and fecal streptococcus data aggregated by stream-stage condition at selected stations, Seco Creek watershed, south-central Texas, during-and-after implementation period of best-management practices

8. Hardness and potassium data aggregated by stream-stage condition at selected stations, Seco Creek watershed, south-central Texas, during-and-after implementation period of best-management practices

9. Dissolved solids and nitrate data aggregated by stream-stage condition at selected stations, Seco Creek watershed, south-central Texas, during-and-after implementation period of best-management practices

\section{TABLES}

1. Rainfall, streamflow, reservoir-content, and water-quality stations in the Seco Creek watershed, southcentral Texas

2-6. Summary statistics for monthly rainfall and streamflow at:

2. Site 4, Seco Creek at Miller Ranch near Utopia, Texas (08201500), during-and-after implementation period of best-management practices

3. Site 6, Seco Creek Reservoir Inflow near Utopia, Texas (08202450), during-and-after implementation period of best-management practices

4. Site 12, Parkers Creek Reservoir Inflow near D’Hanis, Texas (08202790), during-and-after implementation period of best-management practices

5. Site 16, Seco Creek at Rowe Ranch near D’Hanis, Texas (08202700), during-and-after implementation period of best-management practices

6. Site 18, Seco Creek near Yancey, Texas (08202900), during-and-after implementation period of best-management practices 
7-11. Summary statistics for selected physical properties and water-quality constituents at:

7. Site 4, Seco Creek at Miller Ranch near Utopia, Texas (08201500), before-implementation and during-and-after implementation periods of best-management practices

8. Site 6, Seco Creek Reservoir Inflow near Utopia, Texas (08202450), during-and-after implementation period of best-management practices

9. Site 12, Parkers Creek Reservoir Inflow near D'Hanis, Texas (08202790), during-and-after implementation period of best-management practices

10. Site 16, Seco Creek at Rowe Ranch near D'Hanis, Texas (08202700), during-and-after implementation period of best-management practices

11. Site 18, Seco Creek near Yancey, Texas (08202900), during-and-after implementation period of best-management practices 


\title{
Summary Statistics and Graphical Comparisons of Historical Hydrologic and Water-Quality Data, Seco Creek Watershed, South-Central Texas
}

\author{
By David S. Brown, Richard N. Slattery, and Jon R. Gilhousen
}

\section{Abstract}

The U.S. Geological Survey collected hydrologic (rainfall, streamflow, and reservoir content) and water-quality data in the Seco Creek watershed, south-central Texas. Most of the data from 15 sites were collected as part of a study in cooperation with the U.S. Department of Agriculture and the Texas State Soil and Water Conservation Board to evaluate the effects of agricultural best-management practices on surfaceand ground-water quantity and quality in the 255-square-mile watershed. Nearly 400 bestmanagement practices at 58 sites were implemented by landowners in the watershed during March 1990-September 1995.

Most of the data are from the early 1990s, the period during and after implementation of bestmanagement practices. Data from five sites include water quality and are summarized in tables and graphics in the text; and data from all 15 sites are summarized on a diskette.

Maximum annual rainfall among the sites for which data are presented in the text (excluding one site) for the during-and-after-implementation period (March 1990-September 1995) was 53.27 inches in water year 1992. Maximum annual total streamflow among the sites for the period was 63,400 acre-feet, also in water year 1992. At the one site with water-quality data (under base-flow conditions) for both the before-implementation period and the during-and-after implementation period of best-management practices, percentiles $(5,25,50,75,95)$ for specific conductance, nitrate concentration, and fecal coliform density were less for the during-and-after-implementation period than for the before-implementation period.

\section{INTRODUCTION}

The U.S. Geological Survey (USGS), in cooperation with the U.S. Department of Agriculture and the Texas State Soil and Water Conservation Board, began a study in 1990 to evaluate the effects that agricultural best-management practices (BMPs) have on surface- and ground-water quantity and quality in the Seco Creek watershed. The USGS study is part of a larger study known as the Seco Creek Water-Quality Demonstration Project, which began in spring 1990 and is currently (1998) ongoing. The Seco Creek WaterQuality Demonstration Project is intended to demonstrate and transfer technology to farmers and ranchers and thereby encourage the implementation of practices that will protect surface- and ground-water quality within and downstream of the Seco Creek watershed.

BMPs implemented in this study were designed by a number of local, State, and Federal (other than USGS) agencies to (1) reduce the impacts of nonpointsource water pollution from agricultural chemicals, animal wastes, and sediment; (2) suppress erosion and reduce sedimentation; (3) improve ground-water and downstream surface-water quantity and quality; and (4) improve the quality and availability of vegetative cover. Nearly 400 BMPs at 58 sites were implemented by landowners in the Seco Creek watershed during March 1990-September 1995. The primary BMPs applied to land within the watershed include prescribed burning, range seeding, mechanical brush management, alternative chemical brush management, wildlife habitat management, riparian area management, and grazing management. Cropland BMPs include nutrient management, irrigation water management, and integrated crop management (Steffens and Wright, 1995, p. 18). Within the Seco Creek watershed, ranching and farming account for more than 99 percent of the land use. Land uses within the watershed, with the percentage of land that each use represents and percentage of each land-use 
area to which BMPs have been applied, are presented below.

\begin{tabular}{lcc}
\hline Land use & $\begin{array}{c}\text { Percentage of } \\
\text { watershed area }\end{array}$ & $\begin{array}{c}\text { Percentage of land-use area } \\
\text { to which BMPs applied }\end{array}$ \\
\hline Cropland & 9.3 & 70 \\
Pasture & 2.7 & 92 \\
Rangeland & 87.7 & 80 \\
Urban & .3 & 1 \\
\hline
\end{tabular}

The purpose of this report is to summarize the historical USGS hydrologic (rainfall, streamflow, and reservoir content) and water-quality data collected at sites in the Seco Creek watershed, south-central Texas.

Data from 15 sites in the watershed are included in this report. Data from 5 of the 15 sites comprise rainfall, streamflow, and selected water-quality properties and constituents; data from 9 of the remaining 10 sites are rainfall; and data from 1 site are reservoir content. Data from the five sites with rainfall and streamflow measurements and water-quality samples are summarized in tables and graphics in the text; and data from all 15 sites are summarized on a diskette inside the back cover of the report. (See Appendix - "readme" file for description of data on diskette.) Most of the data from the 15 sites are from the early 1990s, the during-andafter implementation period of BMPs. For the five sites for which data are presented in the text, summary statistics for monthly rainfall and monthly streamflow for the during-and-after implementation period and summary statistics for selected water-quality properties and constituents for the before-implementation period (one site) and the during-and-after-implementation period (all sites) are listed in tables. Daily rainfall for four of the five sites and daily mean streamflow for the five sites for the during-and-after implementation period, and selected water-quality properties and constituents relative to stream-stage conditions for the five sites for the during-and-after-implementation period are shown graphically.

The Seco Creek watershed is approximately 50 miles (mi) west of San Antonio (fig. 1) and comprises parts of Bandera, Medina, and Uvalde Counties. Seco Creek generally flows toward the south and has two primary tributaries within the watershed-Little Seco Creek and Parkers Creek (fig. 2). The southernmost site (site 18) in the Seco Creek watershed has a drainage area of approximately 255 square miles $\left(\mathrm{mi}^{2}\right)$ (table 1 at end of report). The northern part of the Seco Creek watershed is characterized by hills of limestone and shale. In this area, Seco Creek loses a substantial amount of flow crossing the Edwards aquifer recharge zone and becomes dry or flows intermittently immediately downstream of the recharge zone (Land and others, 1983, p. 3). Flow also is regulated by small stock ponds and an Edwards aquifer recharge dam (site 13). The middle and southern parts of the Seco Creek watershed are characterized as relatively flat with alluvial sands and gravels (Maclay and Land, 1988, p. A4).

A network of 15 data-collection sites (table 1) was established in the Seco Creek watershed (fig. 2). Data from sites 4, 6, 12, 16, and 18 were selected to provide an overview of hydrologic and water-quality conditions during the Seco Creek Water-Quality Demonstration Project. The data collected at these sites and presented in the text of this report include rainfall, streamflow, reservoir content, and water quality. Each site is equipped with a data-collection platform (DCP) that records selected data every 15 minutes. Each site also is equipped with a Geostationary Operational Environmental Satellite (GOES) transmitter that transmits the recorded data by way of satellite to a USGS computer every 4 hours and more frequently during storm events.

Rainfall data have been collected at site 4 since 1988, streamflow data have been collected at sites 4 and 16 since 1961, and water-quality data have been collected during base-flow conditions at site 4 since 1970. All other rainfall, streamflow, and stream waterquality stations were installed by the USGS during March 1991 or later. The pre-1990 data provide a basis to evaluate the quantity and quality of surface- and ground-water discharge from the headwaters of the Seco Creek watershed before implementation of BMPs, and serve as a reference for comparison of data collected during and after implementation of BMPs.

Rainfall data were collected at 14 sites using a funnel attached to a standpipe. Rainwater is funneled into the standpipe, measured by a float-and-pulley system, and subsequently recorded by data-logging equipment. Approximately 3.5 to 5 inches (in.) of rain can be measured before the standpipe siphons to empty. Rainfall during siphoning is not recorded by the data-logging equipment. Consequently, individual storms and cumulative rainfall might not have been fully measured. Starting in spring 1994, tipping-bucket rain gages were installed next to the standpipe gages at 7 of the 14 sites. The duplicate rain gages record accurate data during intense rainfall events. Rainfall data 


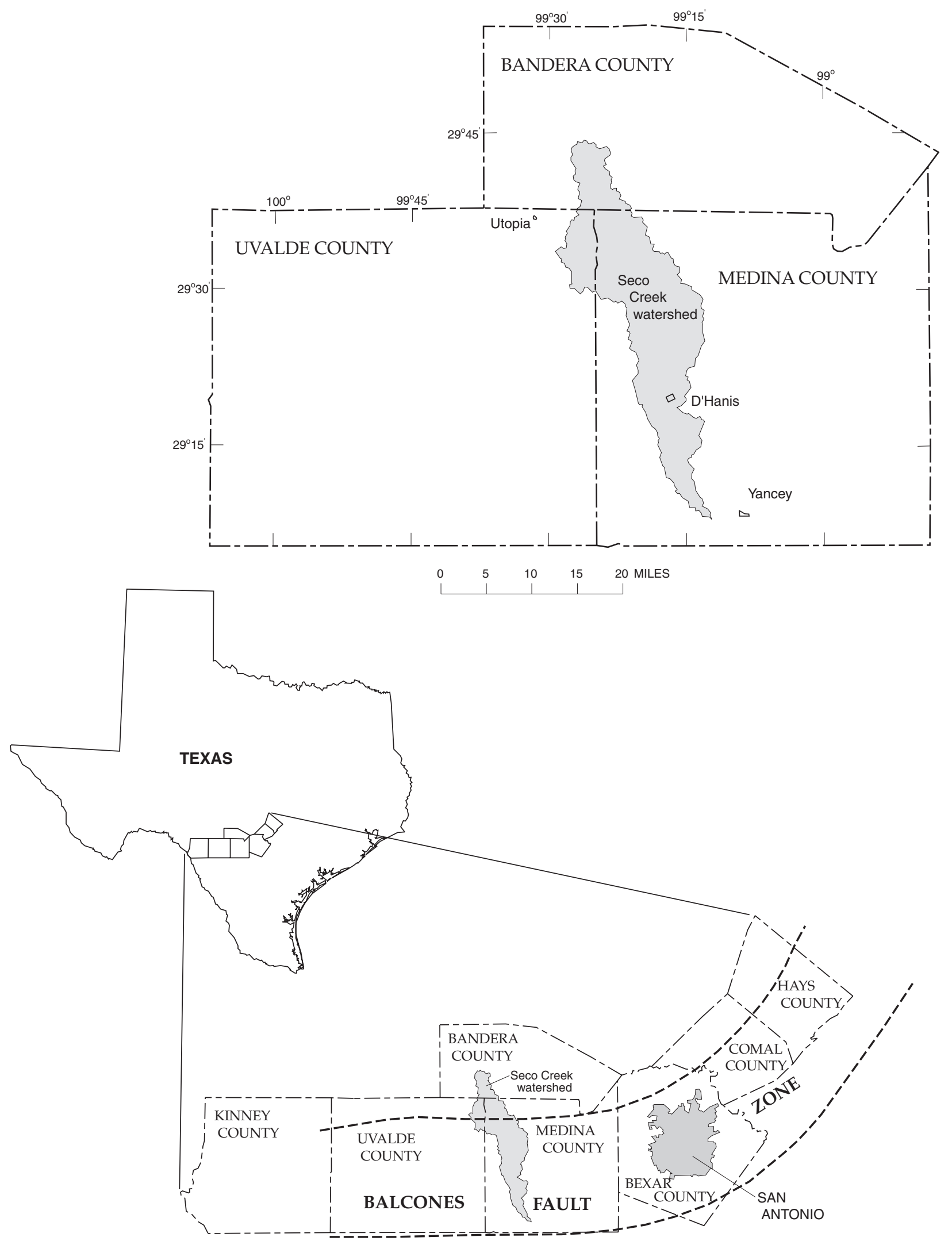

Figure 1. Location of the Seco Creek watershed, south-central Texas. 


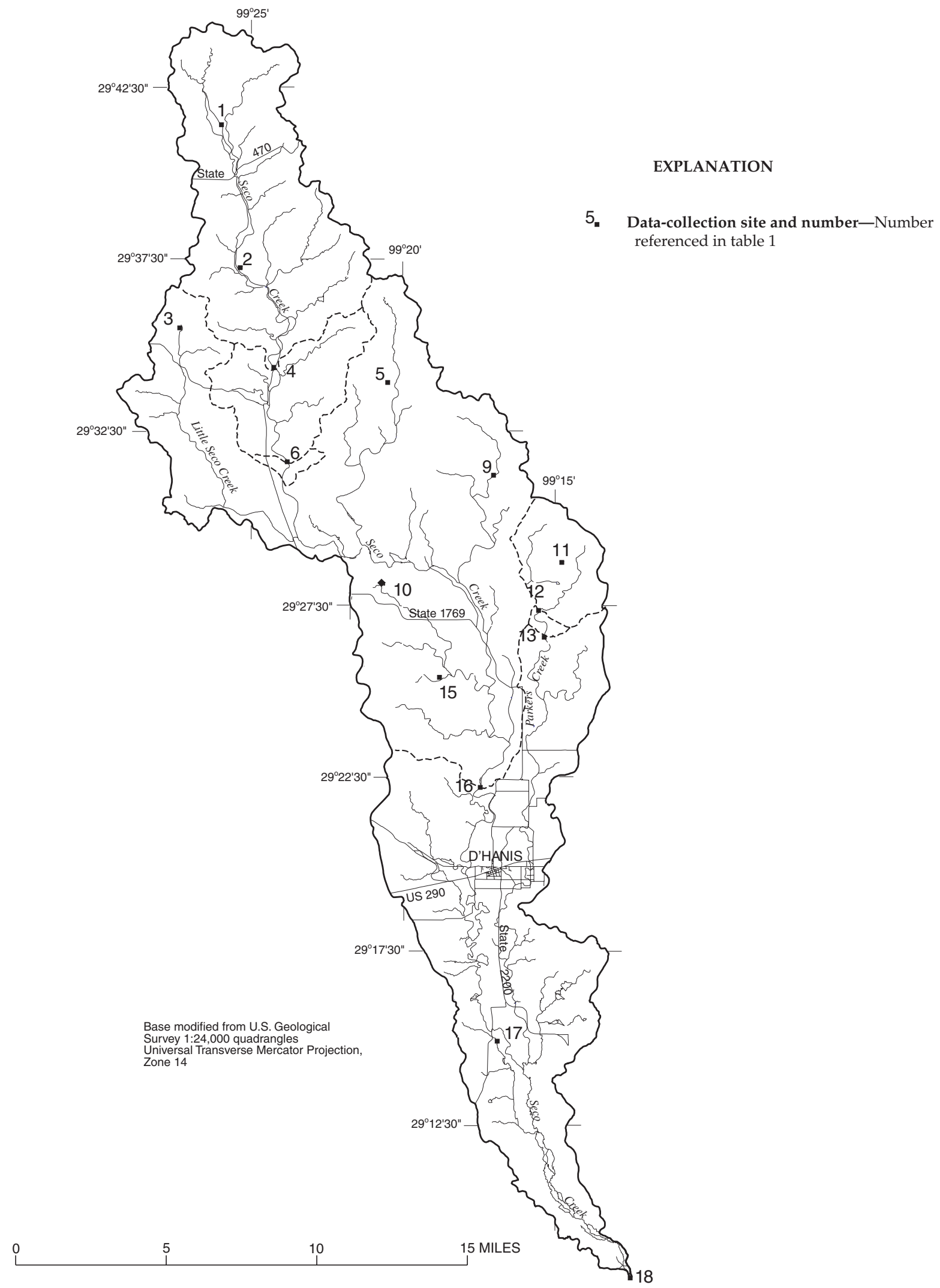

Figure 2. Location of data-collection sites in the Seco Creek watershed, south-central Texas. 
presented in this report are from the standpipe rain gages because a longer period of record is available.

Streamflow data were collected by either a mercury manometer or a pressure transducer gage using carbon dioxide $\left(\mathrm{CO}_{2}\right)$ gas. The $\mathrm{CO}_{2}$ gas flows through a small plastic tube with the open end fixed at a low point in the stream. Changes in stream stage cause changes in back pressure on the $\mathrm{CO}_{2}$ gas, which is measured by the gaging instrument and converted to stage values.

Recorded stage is calibrated to an outside gage located at the site and verified by comparing the recorded data with the observed outside-gage data. Stage data are used in a regression equation to obtain streamflow, which is verified periodically by direct or indirect streamflow measurement.

Discrete water-quality samples were collected manually or by automatic samplers at sites $6,12,16$, and 18 during storm events at intervals of rising, peak, and falling stages.

Quality assurance of data collected from the Seco Creek hydrologic and water-quality data network was accomplished by daily checks of the transmitted DCP data, collection of water-quality equipment blanks and duplicate samples, field verification of stage readings, and measurement of duplicate rain gages at selected stations. Data were reviewed manually and by computerized data-analysis programs.

\section{SUMMARY STATISTICS AND GRAPHICAL COMPARISONS}

Descriptions in this section pertain only to the 5 sites for which data are presented in the text, not to the entire group of 15 sites, unless otherwise noted.

\section{Rainfall and Streamflow}

Daily rainfall for sites 4, 6, 12, and 18 for October 1991 through September 1995 (water years 1992-95), which represents the during-and-after implementation period of BMPs, is shown in figure 3. Daily rainfall for site 16 is omitted because data collection at that site did not begin until June 1994. Monthly rainfall for the five sites for October 1991 (June 1994 for site 16) through September 1995 is listed in tables 2-6 (at end of report). Maximum water year total rainfall among the sites (excluding site 16) for the period was 53.27 in. at site 6 during water year 1992. Maximum monthly rainfall was 10.17 in. at site 6 during December 1991; minimum monthly rainfall was zero at sites 6 and 18 during July
1993. Maximum recorded daily rainfall was $4.55 \mathrm{in}$. at site 6 on September 20, 1995.

Daily mean streamflow for sites 4, 6, 12, 16, and 18 for October 1991 through September 1995 is shown in figure 4. Monthly streamflow for the five sites for that period is listed in tables 2-6. Maximum water year total streamflow among the sites for the period was 63,400 acre-feet (acre-ft) at site 4 during water year 1992. Maximum monthly total streamflow was 14,800 acre-ft at site 4 during December 1991; minimum monthly total streamflow was zero during numerous months at all sites except site 4 , which had perennial flow.

\section{Water Quality}

Water-quality data have been collected during base-flow conditions at site 4 since March 1970 and during stormflow conditions at sites $6,12,16$, and 18 since September 1991.

Graphs of selected water-quality properties and constituents grouped according to six stream-stage (hydrologic) conditions at the five sites for the duringand-after implementation period of BMPs are shown in figures 5-9. The six stream-stage conditions are

\begin{tabular}{|c|c|}
\hline $\begin{array}{l}1 \text { Stable, low } \\
\text { stage }\end{array}$ & $\begin{array}{l}\text { Stable stage when streamflow less than } \\
2.10 \text { cubic feet per second }\left(\mathrm{ft}^{3} / \mathrm{s}\right) \text { during } \\
\text { sampling. }\end{array}$ \\
\hline $\begin{array}{l}2 \text { Stable, } \\
\text { normal } \\
\text { stage }\end{array}$ & $\begin{array}{l}\text { Stable stage when streamflow equal to or } \\
\text { greater than } 2.10 \mathrm{ft}^{3} / \mathrm{s} \text { and less than or equal } \\
\text { to } 17.4 \mathrm{ft}^{3} / \mathrm{s} \text { during sampling. }\end{array}$ \\
\hline $\begin{array}{l}3 \text { Stable, high } \\
\text { stage }\end{array}$ & $\begin{array}{l}\text { Stable stage when streamflow greater than } \\
17.4 \mathrm{ft}^{3} / \mathrm{s} \text { during sampling. }\end{array}$ \\
\hline $\begin{array}{l}4 \text { Rising } \\
\text { stage }\end{array}$ & $\begin{array}{l}\text { Increasing stage associated with storm event } \\
\text { during sampling. }\end{array}$ \\
\hline 5 Peak stage & $\begin{array}{l}\text { Maximum stage associated with storm event } \\
\text { during sampling. }\end{array}$ \\
\hline $\begin{array}{l}6 \text { Falling } \\
\text { stage }\end{array}$ & $\begin{array}{l}\text { Decreasing stage associated with storm event } \\
\text { during sampling. }\end{array}$ \\
\hline
\end{tabular}

Stream-stage conditions 1, 2, and 3 are base-flow conditions and apply only to site 4 . The conditiondelineating flows, 2.10 and $17.4 \mathrm{ft}^{3} / \mathrm{s}$, are the 25 th and 75 th percentiles of the daily mean streamflow at site 4 for the period of record 1961-95. Stream-stage conditions 4, 5, and 6 are stormflow conditions and apply only to sites $6,12,16$, and 18 .

Summary statistics of selected water-quality properties and constituents for the five sites are listed in 

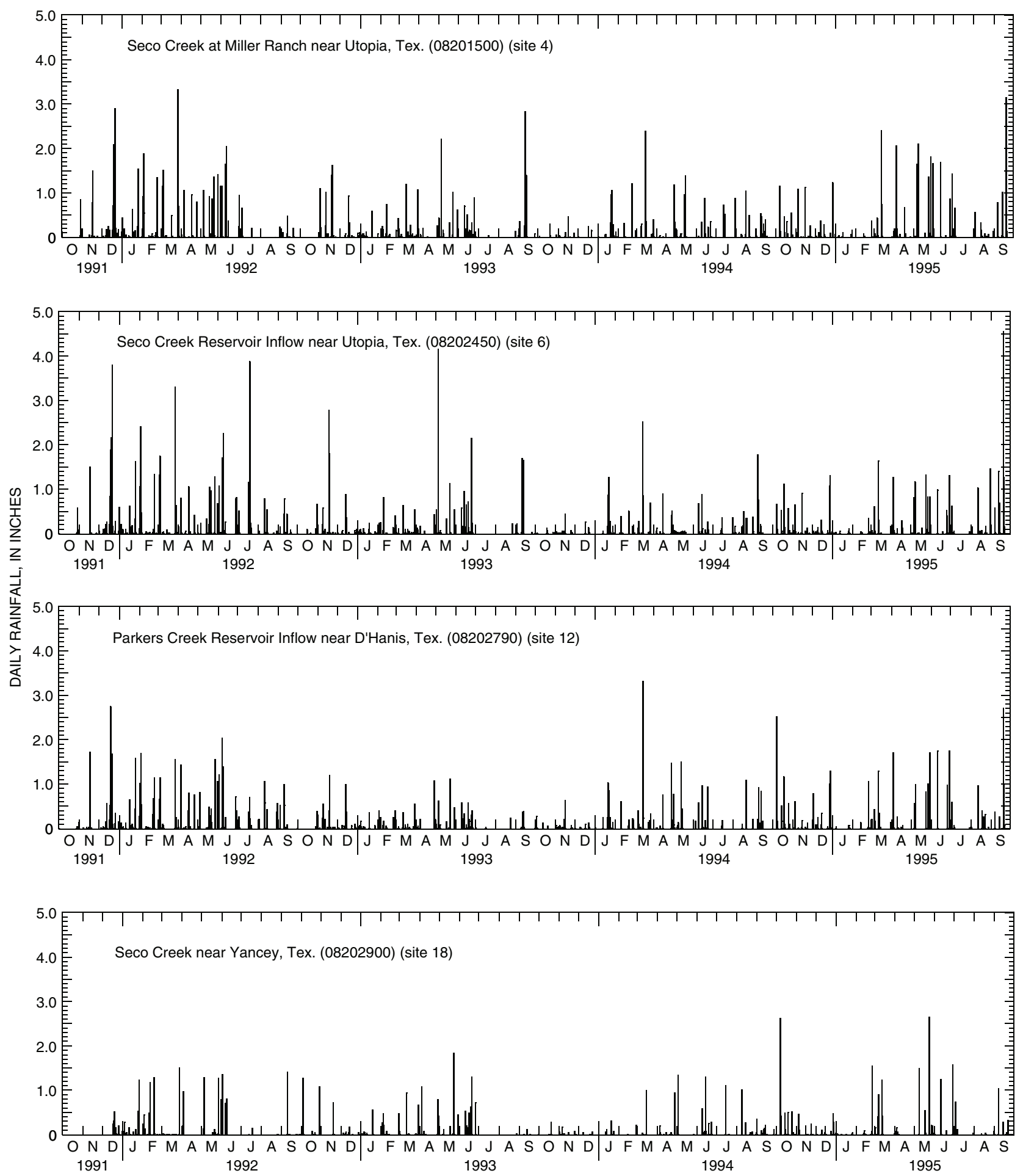

Figure 3. Hydrographs showing daily rainfall at selected stations, Seco Creek watershed, south-central Texas, during-and-after implementation period of best-management practices. 


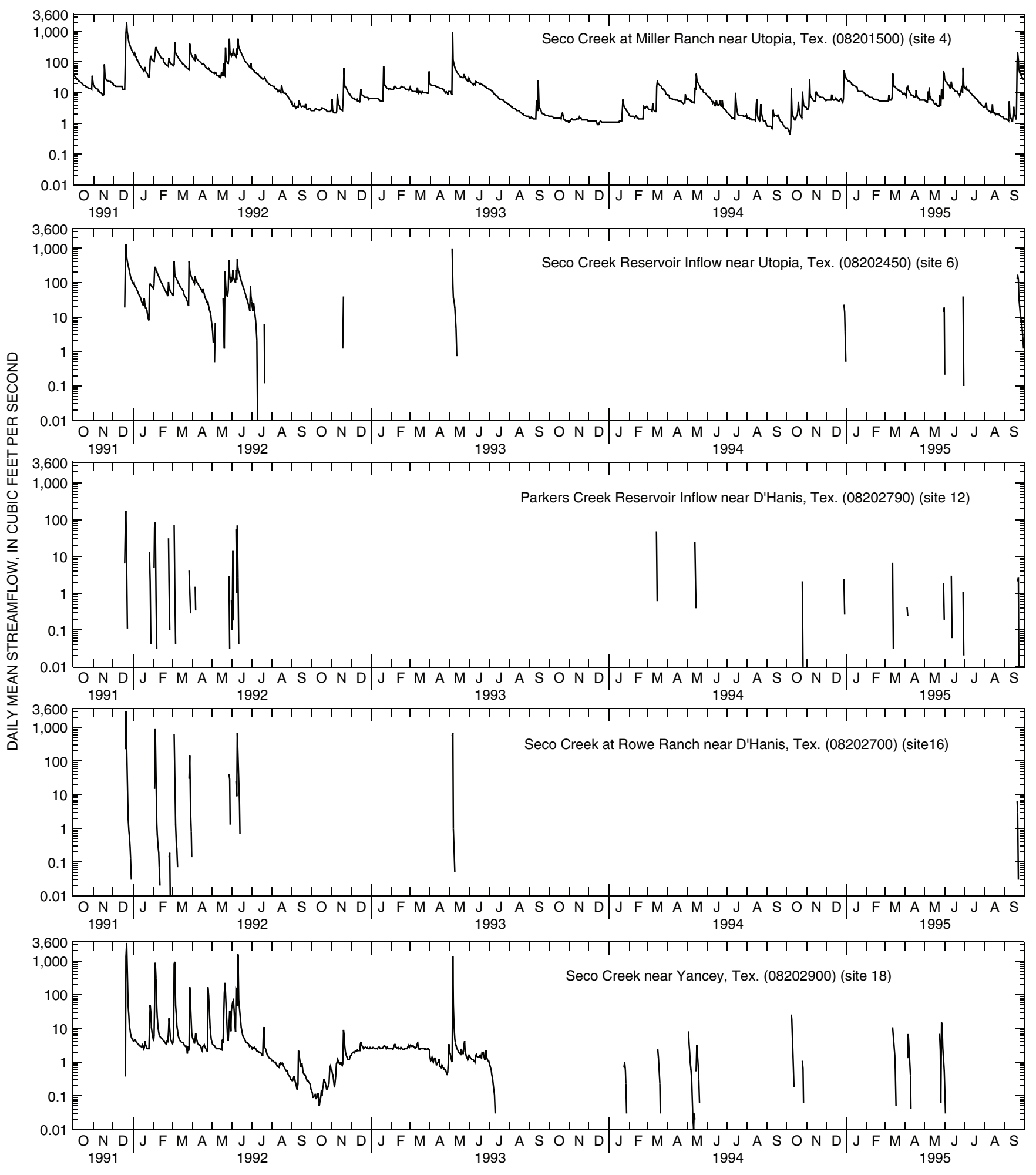

Figure 4. Hydrographs showing daily mean streamflow at selected stations, Seco Creek watershed, south-central Texas, during-and-after implementation period of best-management practices. 

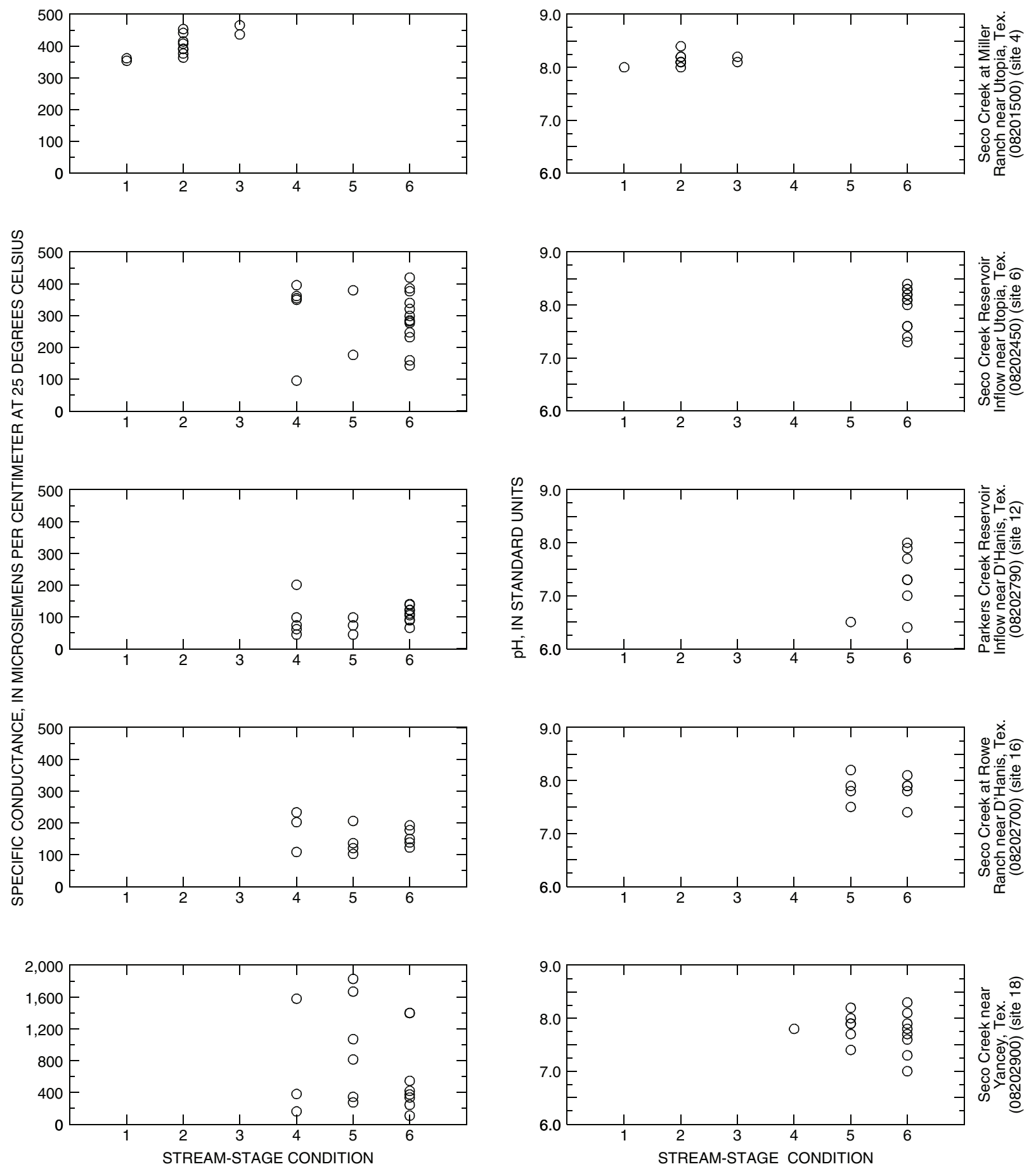

Stream-stage condition: 1-stable, low stage; 2-stable, normal stage; 3-stable, high stage; 4-rising stage; 5-peak stage; 6-falling stage. (1-3 pertain to site $4 ; 4-6$ pertain to sites $6,12,16$, and 18)

Figure 5. Specific conductance and pH data aggregated by stream-stage condition at selected stations, Seco Creek watershed, south-central Texas, during-and-after implementation period of best-management practices. 

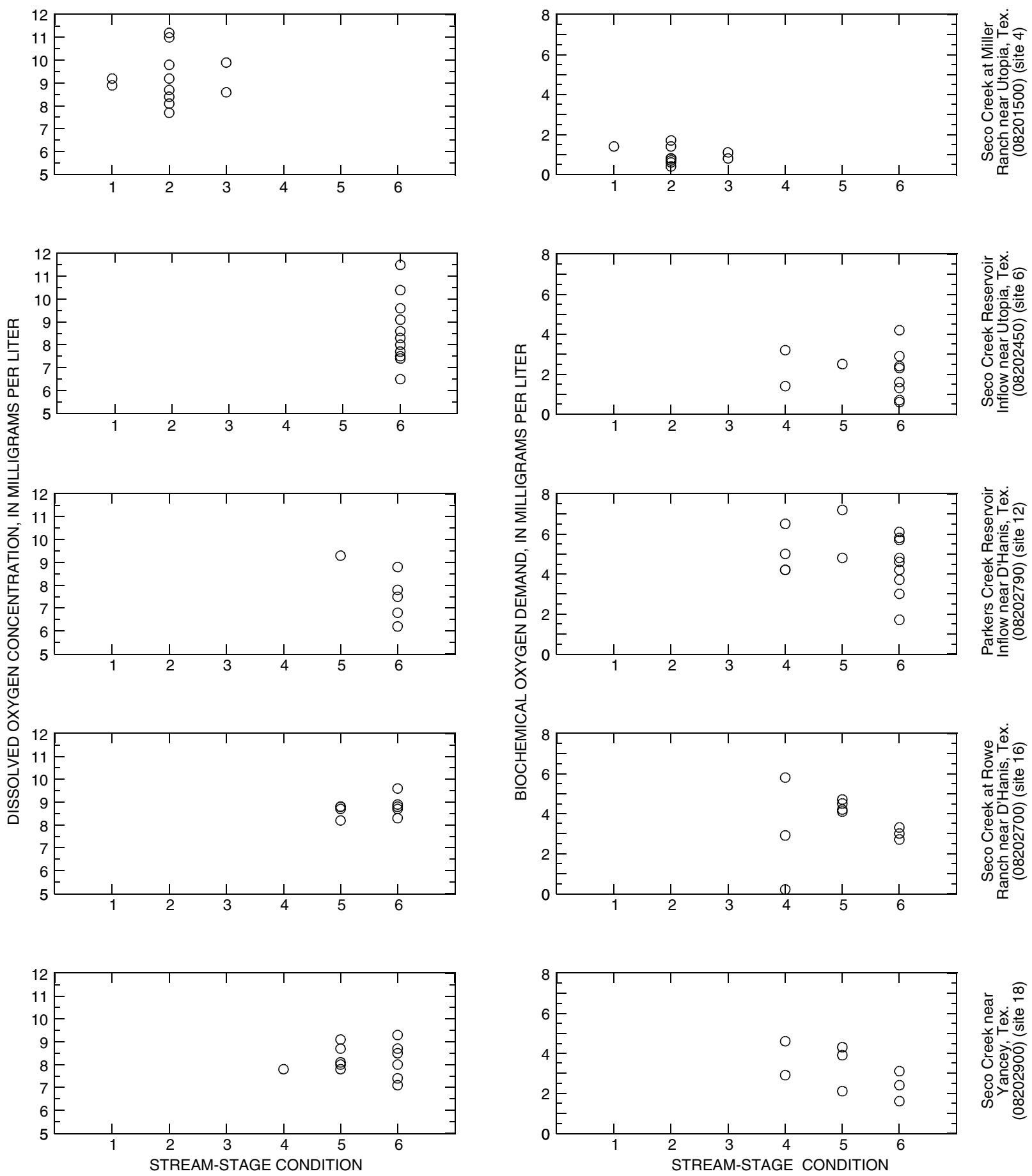

Stream-stage condition: 1-stable, low stage; 2-stable, normal stage; 3-stable, high stage; 4-rising stage; 5-peak stage; 6-falling stage. (1-3 pertain to site $4 ; 4-6$ pertain to sites $6,12,16$, and 18)

Figure 6. Dissolved oxygen and biochemical oxygen demand data aggregated by stream-stage condition at selected stations, Seco Creek watershed, south-central Texas, during-and-after implementation period of bestmanagement practices. 

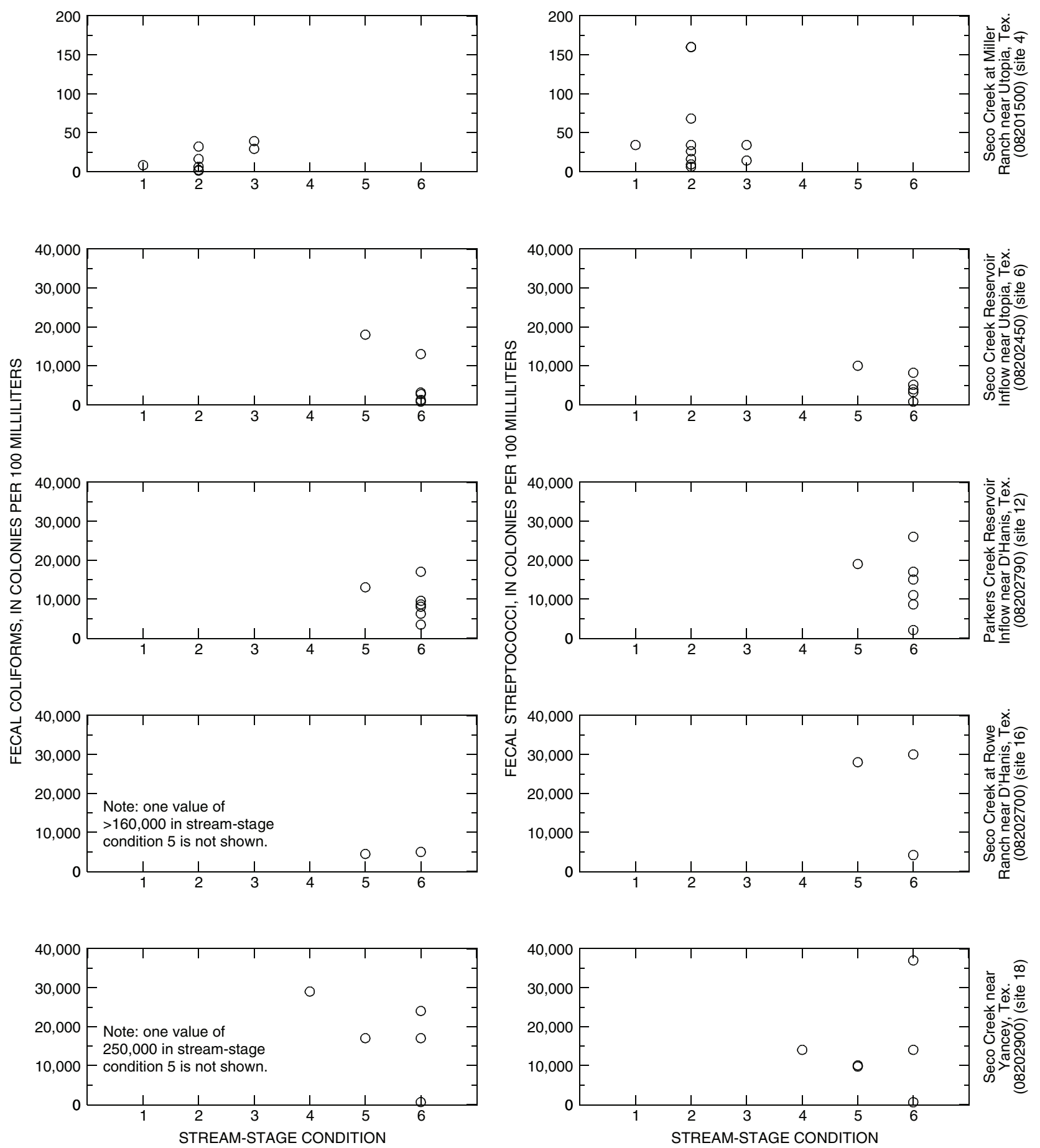

Stream-stage condition: 1-stable, low stage; 2 -stable, normal stage; 3-stable, high stage; 4-rising stage; 5-peak stage; 6-falling stage. (1-3 pertain to site $4 ; 4-6$ pertain to sites $6,12,16$, and 18$)$

Figure 7. Fecal coliform and fecal streptococcus data aggregated by stream-stage condition at selected stations, Seco Creek watershed, south-central Texas, during-and-after implementation period of best-management practices. 

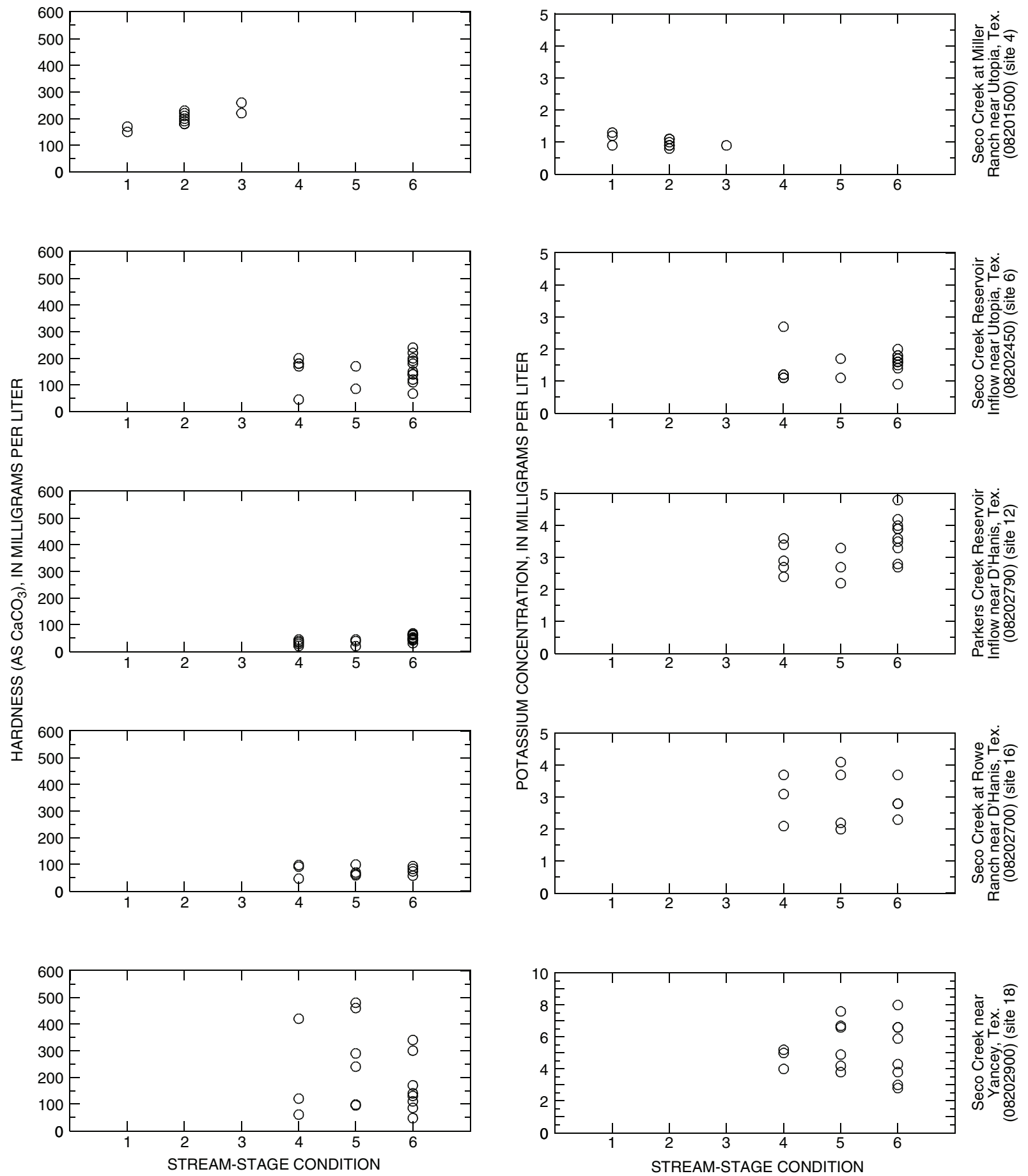

Stream-stage condition: 1-stable, low stage; 2-stable, normal stage; 3-stable, high stage; 4-rising stage; 5-peak stage; 6-falling stage. (1-3 pertain to site $4 ; 4-6$ pertain to sites $6,12,16$, and 18)

Figure 8. Hardness and potassium data aggregated by stream-stage condition at selected stations, Seco Creek watershed, south-central Texas, during-and-after implementation period of best-management practices. 

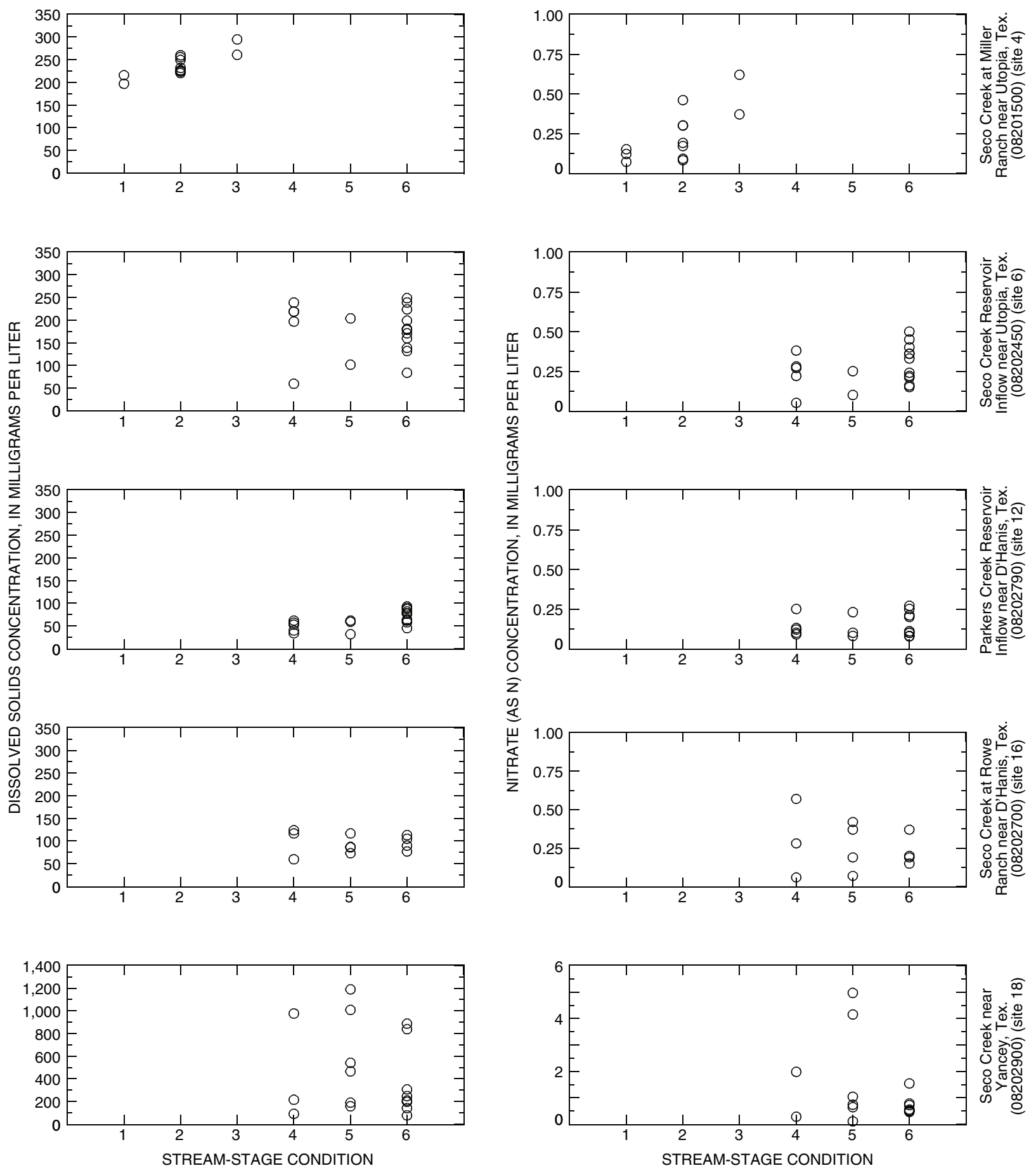

Stream-stage condition: 1-stable, low stage; 2-stable, normal stage; 3-stable, high stage; 4-rising stage; 5-peak stage; 6-falling stage. (1-3 pertain to site $4 ; 4-6$ pertain to sites $6,12,16$, and 18)

Figure 9. Dissolved solids and nitrate data aggregated by stream-stage condition at selected stations, Seco Creek watershed, south-central Texas, during-and-after implementation period of best-management practices. 
tables 7-11 (at end of report). For site 4, the summary statistics are grouped by sampling period-March 1970-February 1990, before-implementation period of BMPs; March 1990-September 1995, during-andafter implementation period of BMPs; and March 1970-September 1995, combined periods. For sites 6, 12,16 , and 18 , the statistics summarize the duringand-after implementation period.

Minimum specific conductance was 44 microsiemens per centimeter at 25 degrees Celsius $(\mu \mathrm{S} / \mathrm{cm})$ at site 12 (table 9), which is on an ephemeral stream with the smallest gaged drainage area of the watershed $\left(9.34 \mathrm{mi}^{2}\right)$ (table 1). Maximum specific conductance was $1,830 \mu \mathrm{S} / \mathrm{cm}$ at site 18 (table 11 ), which is on an intermittent stream with the largest gaged drainage area of the watershed $\left(255 \mathrm{mi}^{2}\right)$. Specific conductances at ephemeral stream sites typically were smaller than at intermittent or perennial stream sites. Specific conductance percentiles at site 4 were slightly smaller for the during-and-after-implementation period than for the before-implementation period (table 7). Specific conductance at site 4 increased slightly from low stage to high stage (fig. 5). Specific conductance typically had a larger range during stormflow conditions than during base-flow conditions; site 18 had the largest range. Unlike site 4, where specific conductance during stable base-flow conditions increased with stage, sites 6,12 , 16 , and 18 showed no apparent relation between specific conductance and stormflow conditions.

Minimum $\mathrm{pH}$ was 6.4 at site 12 (table 9), and maximum $\mathrm{pH}$ was 8.5 at site 4 (before-implementation period) (table 7). Minimum and maximum $\mathrm{pH}$ were similar for all sites, except site 12 , where $\mathrm{pH}$ typically was lower (fig. 5). Site 4 had the smallest $\mathrm{pH}$ range of the five sites for the during-and-after implementation period (fig. 5). $\mathrm{pH}$ percentiles at site 4 were similar for all sampling periods (table 7).

Dissolved oxygen concentrations ranged from a minimum of $6.2 \mathrm{mg} / \mathrm{L}$ at site 12 (table 9) to a maximum of $12.0 \mathrm{mg} / \mathrm{L}$ at site 4 (before-implementation period) (table 7). Dissolved oxygen percentiles at site 4 were similar for all sampling periods (table 7). Dissolved oxygen concentrations at and between sites varied substantially except at site 16 , which had the smallest range in concentration (fig. 6). Dissolved oxygen concentrations showed no apparent relation with the stream-stage conditions during sample collection.

Biochemical oxygen demand (BOD) ranged from a minimum of zero at site 4 (before-implementation period) (table 7) to a maximum of $7.2 \mathrm{mg} / \mathrm{L}$ at site 12 (table 9). Base-flow samples collected at site 4 consistently had the smallest BOD concentrations; larger concentrations were associated with stormflow samples collected at sites 6, 12, 16, and 18 (fig. 6). BOD concentrations decreased between the peak and falling stages at sites 12,16 , and 18 .

A relatively small number of bacteria samples were collected during storm events because of sample processing-time limitations and remoteness of sampling sites. Fecal coliform densities ranged from a minimum of 1 colony per 100 milliliters (cols./100 mL) at site 4 (during-and-after implementation period) (table 7) to a maximum of 250,000 cols. $/ 100 \mathrm{~mL}$ at site 18 (table 11 ). Base-flow samples collected at site 4 consistently had the smallest fecal coliform and fecal streptococcus densities; much larger (typically one to two orders of magnitude) densities were associated with the stormflow samples collected at sites 6, 12, 16, and 18 . Fecal coliform percentiles at site 4 for the during-andafter-implementation period were about $\frac{1}{24}$ to $\frac{1}{3}$ of the percentiles for the before-implementation period (table 7). Fecal streptococcus densities at site 4 generally were smaller for the during-and-after-implementation period than for the before-implementation period.

Hardness ( $\mathrm{as}_{\mathrm{CaCO}}$ ) ranged from a minimum of $19 \mathrm{mg} / \mathrm{L}$ at site 12 (table 9) to a maximum of $480 \mathrm{mg} / \mathrm{L}$ at site 18 (table 11). Hardness percentiles at site 4 were similar for all sampling periods (table 7). Hardness at site 4 increased slightly from low stage to high stage; hardness at sites 12 and 16 was consistently the smallest and had the smallest range (fig. 8).

Dissolved potassium concentrations ranged from a minimum of $0.8 \mathrm{mg} / \mathrm{L}$ at site 4 (table 7 ) to a maximum of $8.0 \mathrm{mg} / \mathrm{L}$ at site 18 (table 11). Larger ranges of potassium concentrations typically were associated with stormwater samples rather than with base-flow samples (fig. 8). Potassium percentiles at site 4 were similar for all sampling periods (table 7). Potassium concentrations for all base-flow and stormwater samples showed no apparent relation with the stream-stage conditions during sample collection (fig. 8).

Dissolved solids concentrations ranged from a minimum of $32 \mathrm{mg} / \mathrm{L}$ at site 12 (table 9 ) to a maximum of $1,190 \mathrm{mg} / \mathrm{L}$ at site 18 (table 11). All dissolved solids percentiles for site 4 except 95 were smaller for the during-and-after-implementation period than for the before-implementation period (table 7). Dissolved solids concentrations at sites 4, 6, 12, and 16 showed little variability (fig. 9). However, site 4 showed a slight increase in dissolved solids concentration from 
low stage to high stage. Site 18 had the largest range of dissolved solids concentrations during stormflow conditions.

Nitrate (as nitrogen) concentrations ranged from a minimum of $0.03 \mathrm{mg} / \mathrm{L}$ at site 4 (beforeimplementation period) (table 7) to a maximum of $4.97 \mathrm{mg} / \mathrm{L}$ at site 18 (table 11). Nitrate percentiles for site 4 were much smaller for the during-andafter-implementation period than for the beforeimplementation period. Nitrate concentrations did not vary substantially at and between sites, except at site 18 , which had the largest range (fig. 9). Nitrate concentrations showed no apparent relation with the stream-stage conditions during sample collection.

\section{REFERENCES CITED}

Land, L.F., Boning, C.W., Harmsen, Lynn, and Reeves, R.D., 1983, Streamflow losses along the Balcones fault zone, Nueces River Basin, Texas: U.S. Geological Survey Water-Resources Investigations Report 83-4168, 72 p.

Maclay, R.W., and Land, L.F., 1988, Simulation of flow in the Edwards aquifer, San Antonio region, Texas, and refinement of storage and flow concepts: U.S. Geological Survey Water-Supply Paper 2336-A, 48 p.

Steffens, Tim, and Wright, Phillip, 1995, Seco Creek WaterQuality Demonstration Project, annual project report fiscal year 1995: U.S. Department of Agriculture, Natural Resources Conservation Service, 254 p. 
Table 1. Rainfall, streamflow, reservoir-content, and water-quality stations in the Seco Creek watershed, southcentral Texas

Streamflow:

PER perennial

INT intermittent

$\mathrm{EPH}$ ephemeral

\section{Type of data:}

rf continuous rainfall, daily sum

sf continuous streamflow, daily mean

qpb periodic base-flow water quality

qps periodic stormflow water quality

rc continuous reservoir content, daily maximum

$\left[\mathrm{mi}^{2}\right.$, square miles; NA, not applicable]

\begin{tabular}{|c|c|c|c|c|c|c|c|c|}
\hline $\begin{array}{c}\text { Site } \\
\text { no. } \\
\text { (fig. 2) }\end{array}$ & $\begin{array}{l}\text { Station } \\
\text { no. }\end{array}$ & Station name & Latitude & Longitude & $\begin{array}{l}\text { Stream- } \\
\text { flow }\end{array}$ & $\begin{array}{c}\text { Drainage } \\
\text { area } \\
\left(\mathrm{mi}^{2}\right)\end{array}$ & $\begin{array}{c}\text { Type } \\
\text { of } \\
\text { data }\end{array}$ & $\begin{array}{l}\text { Period o } \\
\text { record } \\
\text { (water } \\
\text { years) }\end{array}$ \\
\hline 1 & 2941250992554 & Seco Creek rain gage no. 1 & $29^{\circ} 41^{\prime} 25^{\prime \prime}$ & $99^{\circ} 25^{\prime} 54^{\prime \prime}$ & NA & NA & $\mathrm{rf}$ & $1991-95$ \\
\hline 2 & 2937170992513 & Seco Creek rain gage no. 2 & $29^{\circ} 37^{\prime} 17^{\prime \prime}$ & $99^{\circ} 25^{\prime} 13^{\prime \prime}$ & NA & NA & $\mathrm{rf}$ & 1991-95 \\
\hline 3 & 2935500992723 & Freeman rain gage near Utopia, Tex. & $29^{\circ} 35^{\prime} 50^{\prime \prime}$ & $99^{\circ} 27^{\prime} 23^{\prime \prime}$ & NA & NA & $\mathrm{rf}$ & 1994-95 \\
\hline 4 & 08201500 & $\begin{array}{l}\text { Seco Creek at Miller Ranch near } \\
\text { Utopia, Tex. }\end{array}$ & $29^{\circ} 34^{\prime} 23^{\prime \prime}$ & $99^{\circ} 24^{\prime} 10^{\prime \prime}$ & PER & 45.0 & $\begin{array}{l}\mathrm{rf} \\
\mathrm{sf} \\
\mathrm{qpb}\end{array}$ & $\begin{array}{l}1988-95 \\
1961-95 \\
1970-95\end{array}$ \\
\hline 5 & 2933550992019 & Coffee rain gage near Utopia, Tex. & $29^{\circ} 33^{\prime} 55^{\prime \prime}$ & $99^{\circ} 20^{\prime} 19^{\prime \prime}$ & NA & NA & $\mathrm{rf}$ & 1994-95 \\
\hline 6 & 08202450 & $\begin{array}{l}\text { Seco Creek Reservoir Inflow near } \\
\text { Utopia, Tex. }\end{array}$ & $29^{\circ} 31^{\prime} 34^{\prime \prime}$ & $99^{\circ} 23^{\prime} 42^{\prime \prime}$ & INT & 59.4 & $\begin{array}{l}\mathrm{rf} \\
\mathrm{sf} \\
\mathrm{qps}\end{array}$ & $\begin{array}{l}1991-95 \\
1991-95 \\
1991-95\end{array}$ \\
\hline 9 & 2931150991651 & Brown rain gage near D'Hanis, Tex. & $29^{\circ} 31^{\prime} 15^{\prime \prime}$ & $99^{\circ} 16^{\prime} 51^{\prime \prime}$ & NA & NA & $\mathrm{rf}$ & 1995 \\
\hline 10 & 2928080992030 & $\begin{array}{l}\text { Valdina Farms rain gage near } \\
\text { D'Hanis, Tex. }\end{array}$ & $29^{\circ} 28^{\prime} 08^{\prime \prime}$ & $99^{\circ} 20^{\prime} 30^{\prime \prime}$ & NA & NA & $\mathrm{rf}$ & 1994-95 \\
\hline 11 & 2928480991442 & Parkers Creek rain gage no. 1 & $29^{\circ} 28^{\prime} 48^{\prime \prime}$ & $99^{\circ} 14^{\prime} 42^{\prime \prime}$ & NA & NA & $\mathrm{rf}$ & $1991-95$ \\
\hline 12 & 08202790 & $\begin{array}{l}\text { Parkers Creek Reservoir Inflow } \\
\text { near D'Hanis, Tex. }\end{array}$ & $29^{\circ} 27^{\prime} 27^{\prime \prime}$ & $99^{\circ} 15^{\prime} 16^{\prime \prime}$ & $\mathrm{EPH}$ & 9.34 & $\begin{array}{l}\mathrm{rf} \\
\mathrm{sf} \\
\mathrm{qps}\end{array}$ & $\begin{array}{l}1991-95 \\
1991-95 \\
1991-95\end{array}$ \\
\hline 13 & 08202800 & $\begin{array}{l}\text { Parkers Creek Reservoir near } \\
\text { D'Hanis, Tex. }\end{array}$ & $29^{\circ} 26^{\prime} 42^{\prime \prime}$ & $99^{\circ} 15^{\prime} 09^{\prime \prime}$ & $\mathrm{EPH}$ & 10.0 & $\mathrm{rc}$ & $1991-95$ \\
\hline 15 & 2925270991837 & Chaney rain gage near D'Hanis, Tex. & $29^{\circ} 25^{\prime} 27^{\prime \prime}$ & $99^{\circ} 18^{\prime} 37^{\prime \prime}$ & NA & NA & $\mathrm{rf}$ & 1994-95 \\
\hline 16 & 08202700 & $\begin{array}{l}\text { Seco Creek at Rowe Ranch near } \\
\text { D'Hanis, Tex. }\end{array}$ & $29^{\circ} 22^{\prime} 14^{\prime \prime}$ & $99^{\circ} 17^{\prime} 15^{\prime \prime}$ & $\mathrm{EPH}$ & 168 & $\begin{array}{l}\mathrm{rf} \\
\mathrm{sf} \\
\mathrm{qps}\end{array}$ & $\begin{array}{l}1994-95 \\
1961-95 \\
1991-93\end{array}$ \\
\hline 17 & 2914540991642 & Arnold rain gage near D'Hanis, Tex. & $29^{\circ} 14^{\prime} 54^{\prime \prime}$ & $99^{\circ} 16^{\prime} 42^{\prime \prime}$ & NA & NA & $\mathrm{rf}$ & 1994-95 \\
\hline 18 & 08202900 & Seco Creek near Yancey, Tex. & $29^{\circ} 08^{\prime} 03^{\prime \prime}$ & $99^{\circ} 12^{\prime} 19^{\prime \prime}$ & INT & 255 & $\begin{array}{l}\text { rf } \\
\text { sf } \\
\text { qps }\end{array}$ & $\begin{array}{l}1992-95 \\
1991-95 \\
1991-95\end{array}$ \\
\hline
\end{tabular}


$\vec{\sigma} \quad$ Table 2. Summary statistics for monthly rainfall and streamflow at site 4, Seco Creek at Miller Ranch near Utopia, Texas (08201500), during-andafter implementation period of best-management practices

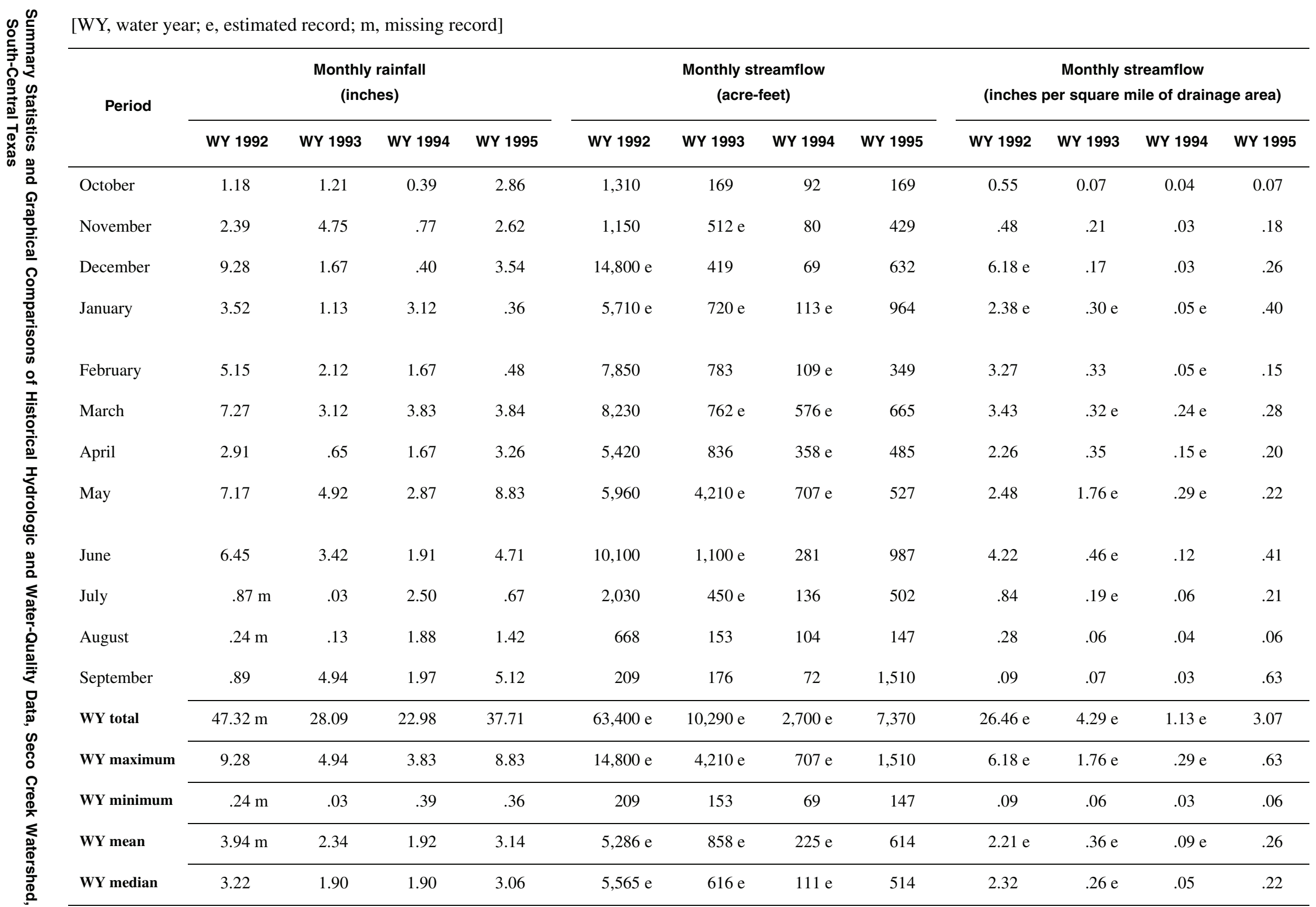


Table 3. Summary statistics for monthly rainfall and streamflow at site 6, Seco Creek Reservoir Inflow near Utopia, Texas (08202450), during-andafter implementation period of best-management practices

[WY, water year; m, missing record; e, estimated record]

\begin{tabular}{|c|c|c|c|c|c|c|c|c|c|c|c|c|}
\hline \multirow{2}{*}{ Period } & \multicolumn{4}{|c|}{$\begin{array}{l}\text { Monthly rainfall } \\
\text { (inches) }\end{array}$} & \multicolumn{4}{|c|}{$\begin{array}{l}\text { Monthly streamflow } \\
\text { (acre-feet) }\end{array}$} & \multicolumn{4}{|c|}{$\begin{array}{c}\text { Monthly streamflow } \\
\text { (inches per square mile of drainage area) }\end{array}$} \\
\hline & WY 1992 & WY 1993 & WY 1994 & WY 1995 & WY 1992 & WY 1993 & WY 1994 & WY 1995 & WY 1992 & WY 1993 & WY 1994 & WY 1995 \\
\hline October & $0.67 \mathrm{~m}$ & 0.87 & 0.24 & 3.24 & 0 & 0 & 0 & 0 & 0 & 0 & 0 & 0 \\
\hline November & $1.52 \mathrm{~m}$ & 5.68 & .77 & 1.90 & 44 & 80 & 0 & 0 & .01 & .03 & 0 & 0 \\
\hline December & 10.17 & 1.58 & .42 & 3.25 & 8,340 & 0 & 0 & 82 & 2.63 & 0 & 0 & .03 \\
\hline January & 4.03 & .83 & 3.05 & .26 & 2,980 & 56 & 0 & 0 & .94 & .02 & 0 & 0 \\
\hline February & 5.60 & 2.05 & 1.07 & .48 & 6,180 & 0 & 0 & 0 & 1.95 & 0 & 0 & 0 \\
\hline March & 7.18 & 1.76 & 4.57 & 2.86 & 6,430 & 36 & 0 & 0 & 2.03 & .01 & 0 & 0 \\
\hline April & 2.56 & .92 & 2.17 & 1.98 & 3,800 & 0 & 0 & 0 & 1.20 & 0 & 0 & 0 \\
\hline May & 4.90 & 6.86 & .67 & 5.24 & $2,990 \mathrm{e}$ & $2,290 \mathrm{e}$ & 9.7 & 65 & $.94 \mathrm{e}$ & $.72 \mathrm{e}$ & 0 & .02 \\
\hline June & 7.03 & 5.99 & 2.19 & 3.25 & $6,710 \mathrm{e}$ & 0 & 0 & 78 & $2.11 \mathrm{e}$ & 0 & 0 & .02 \\
\hline July & 6.16 & 0 & .89 & .74 & $275 \mathrm{e}$ & 0 & 0 & 0 & $.09 \mathrm{e}$ & 0 & 0 & 0 \\
\hline August & 1.52 & .22 & 2.11 & 2.96 & 0 & 0 & 0 & 0 & 0 & 0 & 0 & 0 \\
\hline September & 1.93 & 3.66 & 2.96 & 8.72 & 0 & 0 & 0 & 1,010 & 0 & 0 & 0 & .32 \\
\hline WY total & $53.27 \mathrm{~m}$ & 30.42 & 21.11 & 34.88 & $37,700 \mathrm{e}$ & $2,460 \mathrm{e}$ & 9.7 & 1,240 & $11.90 \mathrm{e}$ & $.78 \mathrm{e}$ & 0 & 0 \\
\hline WY maximum & 10.17 & 6.86 & 4.57 & 8.72 & 8,340 & 2,290 & 9.7 & 1,010 & 2.63 & $.72 \mathrm{e}$ & 0 & .32 \\
\hline WY minimum & $.67 \mathrm{~m}$ & 0 & .24 & .26 & 0 & 0 & 0 & 0 & 0 & 0 & 0 & 0 \\
\hline WY mean & $4.44 \mathrm{~m}$ & 2.54 & 1.76 & 2.91 & $3,146 \mathrm{e}$ & $205 \mathrm{e}$ & .8 & 103 & $.99 \mathrm{e}$ & $.07 \mathrm{e}$ & 0 & .03 \\
\hline WY median & 4.47 & 1.67 & 1.59 & 2.91 & $2,985 \mathrm{e}$ & 0 & 0 & 0 & $.94 \mathrm{e}$ & 0 & 0 & 0 \\
\hline
\end{tabular}


$\vec{\infty} \quad$ Table 4. Summary statistics for monthly rainfall and streamflow at site 12, Parkers Creek Reservoir Inflow near D'Hanis, Texas (08202790), duringand-after implementation period of best-management practices

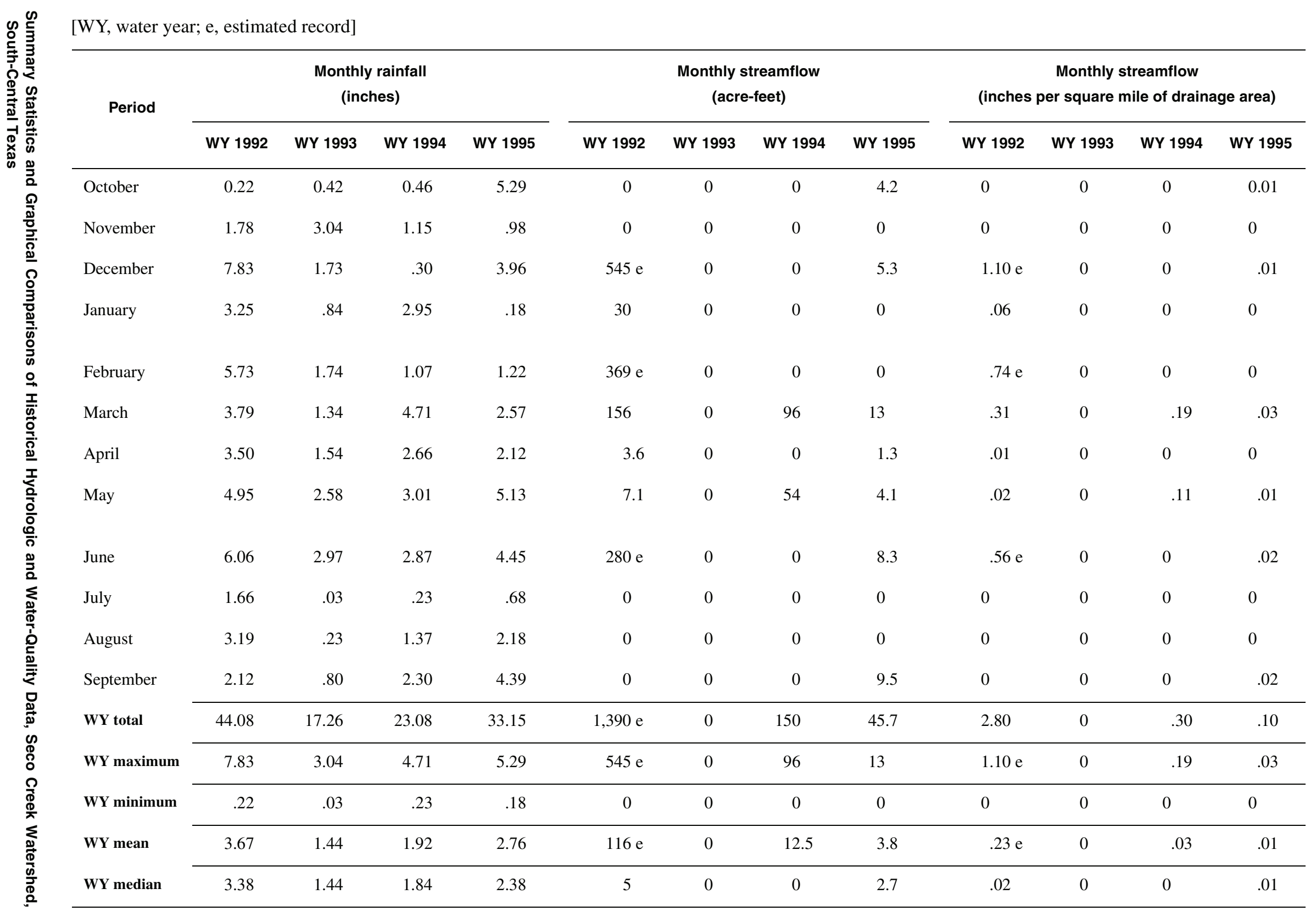


Table 5. Summary statistics for monthly rainfall and streamflow at site 16, Seco Creek at Rowe Ranch near D'Hanis, Texas (08202700), duringand-after implementation period of best-management practices

[WY, water year; --, not collected or computed; e, estimated record; m, missing record]

\begin{tabular}{|c|c|c|c|c|c|c|c|c|c|c|c|c|}
\hline \multirow{2}{*}{ Period } & \multicolumn{4}{|c|}{$\begin{array}{l}\text { Monthly rainfall } \\
\text { (inches) }\end{array}$} & \multicolumn{4}{|c|}{$\begin{array}{l}\text { Monthly streamflow } \\
\text { (acre-feet) }\end{array}$} & \multicolumn{4}{|c|}{$\begin{array}{c}\text { Monthly streamflow } \\
\text { (inches per square mile of drainage area) }\end{array}$} \\
\hline & WY 1992 & WY 1993 & WY 1994 & WY 1995 & WY 1992 & WY 1993 & WY 1994 & WY 1995 & WY 1992 & WY 1993 & WY 1994 & WY 1995 \\
\hline October & -- & -- & -- & 3.12 & 0 & 0 & 0 & 0 & 0 & 0 & 0 & 0 \\
\hline November & -- & -- & -- & .87 & 0 & 0 & 0 & 0 & 0 & 0 & 0 & 0 \\
\hline December & -- & -- & -- & 2.12 & 7,220 & 0 & 0 & 0 & .81 & 0 & 0 & 0 \\
\hline January & -- & -- & -- & .19 & 0 & 0 & 0 & 0 & 0 & 0 & 0 & 0 \\
\hline February & -- & -- & -- & 1.40 & 1,910 & 0 & 0 & 0 & .21 & 0 & 0 & 0 \\
\hline March & -- & -- & -- & 2.40 & 1,660 & 0 & 0 & 0 & .19 & 0 & 0 & 0 \\
\hline April & -- & -- & -- & 1.89 & 0 & 0 & 0 & 0 & 0 & 0 & 0 & 0 \\
\hline May & -- & -- & -- & 3.65 & 141 & $2,490 \mathrm{e}$ & 0 & 0 & .02 & $.28 \mathrm{e}$ & 0 & 0 \\
\hline June & -- & -- & $1.00 \mathrm{~m}$ & 3.52 & $1,820 \mathrm{e}$ & 0 & 0 & 0 & $.20 \mathrm{e}$ & 0 & 0 & 0 \\
\hline July & -- & -- & .53 & .43 & 0 & 0 & 0 & 0 & 0 & 0 & 0 & 0 \\
\hline August & -- & -- & .47 & .58 & 0 & 0 & 0 & 0 & 0 & 0 & 0 & 0 \\
\hline September & -- & -- & 1.29 & 2.42 & 0 & 0 & 0 & 19 & 0 & 0 & 0 & 0 \\
\hline WY total & -- & -- & $3.29 \mathrm{~m}$ & 22.59 & $12,800 \mathrm{e}$ & $2,490 \mathrm{e}$ & 0 & 19 & $1.43 \mathrm{e}$ & $.28 \mathrm{e}$ & 0 & 0 \\
\hline WY maximum & -- & -- & -- & 3.65 & 7,220 & $2,490 \mathrm{e}$ & 0 & 19 & .81 & $.28 \mathrm{e}$ & 0 & 0 \\
\hline WY minimum & -- & -- & -- & .19 & 0 & 0 & 0 & 0 & 0 & 0 & 0 & 0 \\
\hline WY mean & -- & -- & -- & 1.88 & $1,063 \mathrm{e}$ & $208 \mathrm{e}$ & 0 & 1.6 & $.12 \mathrm{e}$ & $.02 \mathrm{e}$ & 0 & 0 \\
\hline WY median & -- & -- & -- & 1.89 & 0 & 0 & 0 & 0 & 0 & 0 & 0 & 0 \\
\hline
\end{tabular}


ح Table 6. Summary statistics for monthly rainfall and streamflow at site 18, Seco Creek near Yancey, Texas (08202900), during-and-after implementation period of best-management practices

\begin{tabular}{|c|c|c|c|c|c|c|c|c|c|c|c|c|}
\hline \multirow{2}{*}{ Period } & \multicolumn{4}{|c|}{$\begin{array}{l}\text { Monthly rainfall } \\
\text { (inches) }\end{array}$} & \multicolumn{4}{|c|}{$\begin{array}{l}\text { Monthly streamflow } \\
\quad \text { (acre-feet) }\end{array}$} & \multicolumn{4}{|c|}{$\begin{array}{c}\text { Monthly streamflow } \\
\text { (inches per square mile of drainage area) }\end{array}$} \\
\hline & WY 1992 & WY 1993 & WY 1994 & WY 1995 & WY 1992 & WY 1993 & WY 1994 & WY 1995 & WY 1992 & WY 1993 & WY 1994 & WY 1995 \\
\hline October & -- & 2.50 & 0.29 & 4.72 & 0.08 & 12 & 0 & 103 & 0 & 0 & 0 & 0.01 \\
\hline November & -- & .74 & .42 & 1.01 & .5 & 89 & 0 & 0 & 0 & .01 & 0 & 0 \\
\hline December & $1.67 \mathrm{~m}$ & .42 & .11 & 1.30 & 10,400 & 151 & 0 & 0 & .77 & .01 & 0 & 0 \\
\hline January & 2.58 & .77 & .83 & .13 & 383 & 163 & $5.1 \mathrm{e}$ & 0 & .03 & .01 & 0 & 0 \\
\hline February & 3.99 & 1.52 & .23 & 1.79 & $2,860 \mathrm{e}$ & 148 & .2 & 0 & $.21 \mathrm{e}$ & .01 & 0 & 0 \\
\hline March & 1.58 & 1.76 & 1.08 & 3.13 & 4,380 & 166 & 9.5 & 46 & .32 & .01 & 0 & 0 \\
\hline April & 1.17 & 2.43 & 1.31 & .29 & 795 & 59 & 0 & $25 \mathrm{e}$ & .06 & 0 & 0 & $0 \mathrm{e}$ \\
\hline May & 3.69 & 2.60 & 1.34 & 5.13 & $1,320 \mathrm{e}$ & 3,040 & 44 & $77 \mathrm{e}$ & $.10 \mathrm{e}$ & .23 & 0 & $.01 \mathrm{e}$ \\
\hline June & 2.89 & 4.34 & 2.62 & 2.92 & 4,770 & 84 & 0 & $2.8 \mathrm{e}$ & .35 & .01 & 0 & $0 \mathrm{e}$ \\
\hline July & $.14 \mathrm{~m}$ & 0 & 1.11 & .89 & 156 & 8.5 & 0 & .1 & .01 & 0 & 0 & 0 \\
\hline August & .01 & .02 & 1.43 & .14 & 46 & 0 & 0 & 0 & 0 & 0 & 0 & 0 \\
\hline September & 1.43 & .13 & 1.07 & 1.93 & 30 & 0 & 0 & 0 & 0 & 0 & 0 & 0 \\
\hline WY total & $19.15 \mathrm{~m}$ & 17.23 & 11.84 & 23.38 & $25,100 \mathrm{e}$ & 3,920 & $58.8 \mathrm{e}$ & $254 \mathrm{e}$ & $1.85 \mathrm{e}$ & .29 & 0 & $.02 \mathrm{e}$ \\
\hline WY maximum & $3.99 \mathrm{~m}$ & 4.34 & 2.62 & 5.13 & 10,400 & 3,040 & 44 & 103 & .77 & .23 & 0 & $.01 \mathrm{e}$ \\
\hline WY minimum & $.01 \mathrm{~m}$ & 0 & .11 & .13 & 0 & 0 & 0 & 0 & 0 & 0 & 0 & 0 \\
\hline WY mean & $1.92 \mathrm{~m}$ & 1.44 & .99 & 1.95 & $2,100 \mathrm{e}$ & 327 & $4.9 \mathrm{e}$ & $21 \mathrm{e}$ & $.15 \mathrm{e}$ & .02 & 0 & $0 \mathrm{e}$ \\
\hline WY median & $1.63 \mathrm{~m}$ & 1.15 & 1.08 & 1.55 & 589 & 86 & 0 & 0 & .05 & .01 & 0 & 0 \\
\hline
\end{tabular}


Table 7. Summary statistics for selected physical properties and water-quality constituents at site 4, Seco Creek at Miller Ranch near Utopia, Texas (08201500), before-implementation and during-and-after implementation periods of best-management practices

Sampling period: 1, March 1970-February 1990, before-implementation period of best-management practices

2, March 1990-September 1995, during-and-after implementation period of best-management practices

3, March 1970-September 1995, combined periods

[Data collected during base-flow conditions. $\mathrm{ft}^{3} / \mathrm{s}$, cubic feet per second; $\mu \mathrm{S} / \mathrm{cm}$, microsiemens per centimeter at 25 degrees Celsius; mg/L, milligrams per liter; $\mathrm{BOD}$, biochemical oxygen demand; cols./100 mL, colonies per 100 milliliters; $\mathrm{CaCO}_{3}$, calcium carbonate; $\mathrm{N}$, nitrogen; <, less than; --, not computed; $\mu \mathrm{g} / \mathrm{L}$, micrograms per liter]

\begin{tabular}{|c|c|c|c|c|c|c|c|c|c|c|}
\hline \multirow{2}{*}{$\begin{array}{l}\text { Selected property } \\
\text { or constituent }\end{array}$} & \multirow{2}{*}{$\begin{array}{l}\text { Sampling } \\
\text { period }\end{array}$} & \multirow{2}{*}{$\begin{array}{c}\text { No. of } \\
\text { samples }\end{array}$} & \multirow{2}{*}{$\begin{array}{l}\text { Maxi- } \\
\text { mum }\end{array}$} & \multirow{2}{*}{$\begin{array}{l}\text { Mini- } \\
\text { mum }\end{array}$} & \multirow{2}{*}{ Mean } & \multicolumn{5}{|c|}{ Percentiles $^{1}$} \\
\hline & & & & & & 95 & 75 & Median & 25 & 5 \\
\hline \multirow[t]{3}{*}{ Discharge, instantaneous $\left(\mathrm{ft}^{3} / \mathrm{s}\right)$} & 1 & 68 & 107 & 0.29 & 16 & 66 & 21 & 7.4 & 2.6 & 0.50 \\
\hline & 2 & 13 & 103 & 1.3 & 16 & 103 & 14 & 7.0 & 2.4 & 1.3 \\
\hline & 3 & 81 & 107 & .29 & 16 & 69 & 20 & 7.4 & 2.6 & .54 \\
\hline \multirow[t]{3}{*}{ Specific conductance $(\mu \mathrm{S} / \mathrm{cm})$} & 1 & 67 & 516 & 356 & 425 & 490 & 450 & 428 & 395 & 368 \\
\hline & 2 & 13 & 466 & 354 & 401 & 466 & 440 & 392 & 363 & 354 \\
\hline & 3 & 80 & 516 & 354 & 421 & 482 & 448 & 423 & 391 & 360 \\
\hline \multirow[t]{3}{*}{$\mathrm{pH}$ (standard units) } & 1 & 67 & 8.5 & 7.4 & 8.1 & 8.5 & 8.2 & 8.0 & 7.9 & 7.6 \\
\hline & 2 & 13 & 8.4 & 8.0 & 8.1 & 8.4 & 8.2 & 8.1 & 8.0 & 8.0 \\
\hline & 3 & 80 & 8.5 & 7.4 & 8.1 & 8.4 & 8.2 & 8.1 & 8.0 & 7.7 \\
\hline \multirow[t]{3}{*}{ Dissolved oxygen (mg/L) } & 1 & 65 & 12.0 & 7.4 & 9.5 & 11.3 & 10.2 & 9.6 & 8.4 & 7.5 \\
\hline & 2 & 13 & 11.2 & 7.7 & 9.2 & 11.2 & 9.8 & 8.9 & 8.5 & 7.7 \\
\hline & 3 & 78 & 12.0 & 7.4 & 9.4 & 11.2 & 10.2 & 9.4 & 8.4 & 7.7 \\
\hline \multirow[t]{3}{*}{ BOD, 5-day (mg/L) } & 1 & 64 & 1.6 & 0 & .5 & 1.5 & .7 & .4 & .2 & 0 \\
\hline & 2 & 11 & 1.7 & .4 & 1.0 & 1.7 & 1.4 & .8 & .7 & .4 \\
\hline & 3 & 75 & 1.7 & 0 & .6 & 1.5 & .8 & .5 & .3 & .1 \\
\hline \multirow[t]{3}{*}{ Coliforms, fecal (cols./100 mL) } & 1 & 45 & 1,800 & 2 & 180 & 940 & 200 & 41 & 16 & 3 \\
\hline & 2 & 10 & 39 & 1 & 14 & 39 & 30 & 7 & 2 & 1 \\
\hline & 3 & 55 & 1,800 & 1 & 150 & 900 & 150 & 32 & 8 & 2 \\
\hline \multirow[t]{3}{*}{ Streptococci, fecal (cols./100 mL) } & 1 & 46 & 580 & 1 & 87 & 490 & 88 & 44 & 18 & 5 \\
\hline & 2 & 11 & 160 & 6 & 51 & 160 & 68 & 34 & 14 & 6 \\
\hline & 3 & 57 & 580 & 1 & 80 & 350 & 84 & 34 & 16 & 6 \\
\hline \multirow[t]{3}{*}{ Hardness, total as $\mathrm{CaCO}_{3}(\mathrm{mg} / \mathrm{L})$} & 1 & 66 & 250 & 160 & 210 & 250 & 220 & 210 & 190 & 170 \\
\hline & 2 & 13 & 260 & 150 & 200 & 260 & 220 & 190 & 180 & 150 \\
\hline & 3 & 79 & 260 & 150 & 200 & 250 & 220 & 200 & 190 & 170 \\
\hline \multirow[t]{3}{*}{ Calcium, dissolved (mg/L) } & 1 & 66 & 82 & 47 & 63 & 80 & 70 & 62 & 57 & 49 \\
\hline & 2 & 13 & 89 & 46 & 62 & 89 & 71 & 59 & 54 & 46 \\
\hline & 3 & 79 & 89 & 46 & 63 & 81 & 70 & 62 & 56 & 47 \\
\hline \multirow[t]{3}{*}{ Magnesium, dissolved (mg/L) } & 1 & 66 & 14 & 10 & 12 & 13 & 12 & 12 & 11 & 11 \\
\hline & 2 & 13 & 12 & 8.7 & 10 & 12 & 10 & 10 & 9.4 & 8.7 \\
\hline & 3 & 79 & 14 & 8.7 & 11 & 13 & 12 & 11 & 11 & 9.6 \\
\hline \multirow[t]{3}{*}{ Sodium, dissolved (mg/L) } & 1 & 65 & 11 & 5.6 & 7.6 & 9.4 & 8.0 & 7.4 & 7.0 & 6.0 \\
\hline & 2 & 13 & 8.5 & 6.3 & 7.1 & 8.5 & 7.7 & 6.9 & 6.4 & 6.3 \\
\hline & 3 & 78 & 11 & 5.6 & 7.5 & 9.4 & 7.9 & 7.4 & 6.9 & 6.0 \\
\hline
\end{tabular}

Footnote at end of table. 
Table 7. Summary statistics for selected physical properties and water-quality constituents at site 4, Seco Creek at Miller Ranch near Utopia, Texas (08201500), before-implementation and during-and-after implementation periods of best-management practices-Continued

\begin{tabular}{|c|c|c|c|c|c|c|c|c|c|c|}
\hline \multirow{2}{*}{$\begin{array}{l}\text { Selected property } \\
\text { or constituent }\end{array}$} & \multirow{2}{*}{$\begin{array}{l}\text { Sampling } \\
\text { period }\end{array}$} & \multirow{2}{*}{$\begin{array}{l}\text { No. of } \\
\text { samples }\end{array}$} & \multirow{2}{*}{$\begin{array}{l}\text { Maxi- } \\
\text { mum }\end{array}$} & \multirow{2}{*}{$\begin{array}{l}\text { Mini- } \\
\text { mum }\end{array}$} & \multirow{2}{*}{ Mean } & \multicolumn{5}{|c|}{ Percentiles $^{1}$} \\
\hline & & & & & & 95 & 75 & Median & 25 & 5 \\
\hline \multirow[t]{3}{*}{ Potassium, dissolved (mg/L) } & 1 & 65 & 1.7 & .8 & 1.1 & 1.6 & 1.2 & 1.0 & 1.0 & .8 \\
\hline & 2 & 13 & 1.3 & .8 & 1.0 & 1.3 & 1.1 & .9 & .9 & .8 \\
\hline & 3 & 78 & 1.7 & .8 & 1.1 & 1.6 & 1.2 & 1.0 & .9 & .8 \\
\hline \multirow[t]{3}{*}{ Sulfate, dissolved (mg/L) } & 1 & 66 & 79 & 25 & 46 & 72 & 55 & 44 & 36 & 29 \\
\hline & 2 & 13 & 51 & 27 & 39 & 51 & 48 & 38 & 32 & 27 \\
\hline & 3 & 79 & 79 & 25 & 45 & 66 & 52 & 44 & 36 & 29 \\
\hline \multirow[t]{3}{*}{ Chloride, dissolved (mg/L) } & 1 & 66 & 18 & 8.3 & 13 & 17 & 15 & 13 & 11 & 8.6 \\
\hline & 2 & 13 & 17 & 9.6 & 12 & 17 & 14 & 11 & 10 & 9.6 \\
\hline & 3 & 79 & 18 & 8.3 & 13 & 17 & 14 & 13 & 11 & 8.9 \\
\hline \multirow{3}{*}{$\begin{array}{l}\text { Dissolved solids, sum of } \\
\text { constituents (mg/L) }\end{array}$} & 1 & 65 & 286 & 208 & 247 & 283 & 264 & 245 & 230 & 216 \\
\hline & 2 & 13 & 295 & 197 & 236 & 295 & 258 & 227 & 218 & 197 \\
\hline & 3 & 78 & 295 & 197 & 245 & 284 & 261 & 245 & 226 & 212 \\
\hline \multirow[t]{3}{*}{ Nitrate, total as N (mg/L) } & 1 & 59 & 1.59 & .03 & .59 & 1.50 & .80 & .53 & .30 & .10 \\
\hline & 2 & 13 & .62 & .07 & .25 & .62 & .34 & .19 & .10 & .07 \\
\hline & 3 & 72 & 1.59 & .03 & .53 & 1.37 & .74 & .47 & .20 & .09 \\
\hline \multirow[t]{3}{*}{ Phosphorus, total (mg/L) } & 1 & 65 & .17 & $<.01$ & -- & .03 & .01 & $<.01$ & $<.01$ & $<.01$ \\
\hline & 2 & 8 & $<.01$ & $<.01$ & $<.01$ & -- & -- & -- & -- & -- \\
\hline & 3 & 73 & .17 & $<.01$ & -- & .03 & .01 & $<.01$ & $<.01$ & $<.01$ \\
\hline \multirow[t]{3}{*}{ Strontium, dissolved $(\mu \mathrm{g} / \mathrm{L})$} & 1 & 9 & 480 & 290 & 410 & -- & -- & -- & -- & -- \\
\hline & 2 & 10 & 480 & 300 & 380 & 480 & 420 & 360 & 330 & 300 \\
\hline & 3 & 19 & 480 & 290 & 390 & 480 & 440 & 400 & 330 & 290 \\
\hline
\end{tabular}

\footnotetext{
${ }^{1}$ The nth percentile is the value exceeded by no more than $n$ percent of the data.
}

Table 8. Summary statistics for selected physical properties and water-quality constituents at site 6, Seco Creek Reservoir Inflow near Utopia, Texas (08202450), during-and-after implementation period of best-management practices

[Data collected during stormflow conditions. $\mathrm{ft}^{3} / \mathrm{s}$, cubic feet per second; $\mu \mathrm{S} / \mathrm{cm}$, microsiemens per centimeter at 25 degrees Celsius; mg/L, milligrams per liter; BOD, biochemical oxygen demand; cols./100 mL, colonies per 100 milliliters; --, not computed; $\mathrm{CaCO}_{3}$, calcium carbonate; $\mathrm{N}$, nitrogen; <, less than; $\mu \mathrm{g} / \mathrm{L}$, micrograms per liter]

\begin{tabular}{|c|c|c|c|c|c|c|c|c|c|}
\hline \multirow{2}{*}{ Selected property or constituent } & \multirow{2}{*}{$\begin{array}{c}\text { No. of } \\
\text { samples }\end{array}$} & \multirow{2}{*}{$\begin{array}{l}\text { Maxi- } \\
\text { mum }\end{array}$} & \multirow{2}{*}{$\begin{array}{l}\text { Mini- } \\
\text { mum }\end{array}$} & \multirow{2}{*}{ Mean } & \multicolumn{5}{|c|}{ Percentiles $^{1}$} \\
\hline & & & & & 95 & 75 & Median & 25 & 5 \\
\hline Discharge, instantaneous $\left(\mathrm{ft}^{3} / \mathrm{s}\right)$ & 26 & 935 & 6.8 & 126 & 762 & 150 & 76 & 24 & 7.7 \\
\hline Specific conductance $(\mu \mathrm{S} / \mathrm{cm})$ & 21 & 420 & 95 & 288 & 418 & 370 & 299 & 204 & 100 \\
\hline pH (standard units) & 13 & 8.4 & 7.3 & 7.9 & 8.4 & 8.2 & 8.1 & 7.5 & 7.3 \\
\hline Dissolved oxygen (mg/L) & 12 & 11.5 & 6.5 & 8.6 & 11.5 & 9.5 & 8.3 & 7.6 & 6.5 \\
\hline BOD, 5-day (mg/L) & 11 & 4.2 & .6 & 2.1 & 4.2 & 2.9 & 2.3 & 1.3 & 6 \\
\hline Coliforms, fecal (cols./100 mL) & 6 & 18,000 & 780 & 6,500 & -- & -- & -- & -- & -- \\
\hline
\end{tabular}

Footnote at end of table. 
Table 8. Summary statistics for selected physical properties and water-quality constituents at site 6 , Seco Creek Reservoir Inflow near Utopia, Texas (08202450), during-and-after implementation period of best-management practices-Continued

\begin{tabular}{|c|c|c|c|c|c|c|c|c|c|}
\hline \multirow{2}{*}{ Selected property or constituent } & \multirow{2}{*}{$\begin{array}{c}\text { No. of } \\
\text { samples }\end{array}$} & \multirow{2}{*}{$\begin{array}{l}\text { Maxi- } \\
\text { mum }\end{array}$} & \multirow{2}{*}{$\begin{array}{l}\text { Mini- } \\
\text { mum }\end{array}$} & \multirow{2}{*}{ Mean } & \multicolumn{5}{|c|}{ Percentiles $^{1}$} \\
\hline & & & & & 95 & 75 & Median & 25 & 5 \\
\hline Streptococci, fecal (cols./100 mL) & 6 & 10,000 & 780 & 5,200 & -- & -- & -- & -- & -- \\
\hline Hardness, total as $\mathrm{CaCO}_{3}(\mathrm{mg} / \mathrm{L})$ & 19 & 240 & 45 & 160 & 240 & 190 & 170 & 120 & 45 \\
\hline Calcium, dissolved (mg/L) & 19 & 86 & 16 & 52 & 86 & 63 & 54 & 39 & 16 \\
\hline Magnesium, dissolved (mg/L) & 19 & 9.7 & 1.1 & 6.3 & 9.7 & 8.4 & 6.6 & 4.9 & 1.1 \\
\hline Sodium, dissolved (mg/L) & 19 & 7.4 & 1.4 & 4.0 & 7.4 & 4.9 & 3.5 & 2.8 & 1.4 \\
\hline Potassium, dissolved (mg/L) & 19 & 2.7 & .9 & 1.5 & 2.7 & 1.7 & 1.6 & 1.2 & .9 \\
\hline Sulfate, dissolved (mg/L) & 19 & 43 & 2.2 & 23 & 43 & 32 & 21 & 12 & 2.2 \\
\hline Chloride, dissolved (mg/L) & 19 & 16 & 1.7 & 6.7 & 16 & 8.1 & 6.2 & 4.1 & 1.7 \\
\hline Dissolved solids, sum of constituents (mg/L) & 18 & 249 & 60 & 178 & 249 & 220 & 189 & 137 & 60 \\
\hline Nitrate, total as N (mg/L) & 19 & .50 & .05 & .27 & .50 & .36 & .25 & .21 & .05 \\
\hline Phosphorus, total (mg/L) & 8 & .08 & $<.01$ & -- & -- & -- & -- & -- & -- \\
\hline Strontium, dissolved ( $\mu \mathrm{g} / \mathrm{L})$ & 19 & 360 & 35 & 240 & 360 & 310 & 250 & 170 & 35 \\
\hline
\end{tabular}

${ }^{1}$ The nth percentile is the value exceeded by no more than $n$ percent of the data.

Table 9. Summary statistics for selected physical properties and water-quality constituents at site 12, Parkers Creek Reservoir Inflow near D'Hanis, Texas (08202790), during-and-after implementation period of bestmanagement practices

[Data collected during stormflow conditions. $\mathrm{ft}^{3} / \mathrm{s}$, cubic feet per second; $\mu \mathrm{S} / \mathrm{cm}$, microsiemens per centimeter at 25 degrees Celsius; --, not computed; mg/L, milligrams per liter; BOD, biochemical oxygen demand; cols./100 mL, colonies per 100 milliliters; $\mathrm{CaCO}_{3}$, calcium carbonate; $\mathrm{N}$, nitrogen; $\mu \mathrm{g} / \mathrm{L}$, micrograms per liter]

\begin{tabular}{|c|c|c|c|c|c|c|c|c|c|}
\hline \multirow{2}{*}{ Selected property or constituent } & \multirow{2}{*}{$\begin{array}{c}\text { No. of } \\
\text { samples }\end{array}$} & \multirow{2}{*}{$\begin{array}{l}\text { Maxi- } \\
\text { mum }\end{array}$} & \multirow{2}{*}{$\begin{array}{l}\text { Mini- } \\
\text { mum }\end{array}$} & \multirow{2}{*}{ Mean } & \multicolumn{5}{|c|}{ Percentiles $^{1}$} \\
\hline & & & & & 95 & 75 & Median & 25 & 5 \\
\hline Discharge, instantaneous $\left(\mathrm{ft}^{3} / \mathrm{s}\right)$ & 25 & 143 & 0.97 & 17 & 124 & 16 & 8.4 & 2.2 & 0.97 \\
\hline Specific conductance $(\mu \mathrm{S} / \mathrm{cm})$ & 18 & 201 & 44 & 98 & 201 & 121 & 94 & 71 & 44 \\
\hline pH (standard units) & 9 & 8.0 & 6.4 & 7.3 & -- & -- & -- & -- & -- \\
\hline Dissolved oxygen (mg/L) & 7 & 9.3 & 6.2 & 7.6 & -- & -- & -- & -- & -- \\
\hline BOD, 5-day (mg/L) & 15 & 7.2 & 1.7 & 4.8 & 7.2 & 5.8 & 4.8 & 4.2 & 1.7 \\
\hline Coliforms, fecal (cols./100 mL) & 7 & 17,000 & 3,400 & 9,400 & -- & -- & -- & -- & -- \\
\hline Streptococci, fecal (cols./100 mL) & 7 & 26,000 & 2,000 & 14,000 & -- & -- & -- & -- & -- \\
\hline Hardness, total as $\mathrm{CaCO}_{3}(\mathrm{mg} / \mathrm{L})$ & 18 & 67 & 19 & 43 & 67 & 54 & 44 & 32 & 19 \\
\hline Calcium, dissolved (mg/L) & 18 & 25 & 6.8 & 16 & 25 & 20 & 16 & 12 & 6.8 \\
\hline Magnesium, dissolved (mg/L) & 18 & 1.3 & .43 & .85 & 1.3 & 1.1 & .81 & .64 & .43 \\
\hline Sodium, dissolved (mg/L) & 18 & 1.4 & .5 & .8 & 1.4 & 1.0 & .8 & .5 & .5 \\
\hline Potassium, dissolved (mg/L) & 18 & 4.8 & 2.2 & 3.3 & 4.8 & 3.9 & 3.4 & 2.7 & 2.2 \\
\hline
\end{tabular}

Footnote at end of table. 
Table 9. Summary statistics for selected physical properties and water-quality constituents at site 12, Parkers Creek Reservoir Inflow near D'Hanis, Texas (08202790), during-and-after implementation period of bestmanagement practices-Continued

\begin{tabular}{|c|c|c|c|c|c|c|c|c|c|}
\hline \multirow{2}{*}{ Selected property or constituent } & \multirow{2}{*}{$\begin{array}{c}\text { No. of } \\
\text { samples }\end{array}$} & \multirow{2}{*}{$\begin{array}{l}\text { Maxi- } \\
\text { mum }\end{array}$} & \multirow{2}{*}{$\begin{array}{l}\text { Mini- } \\
\text { mum }\end{array}$} & \multirow{2}{*}{ Mean } & \multicolumn{5}{|c|}{ Percentiles $^{1}$} \\
\hline & & & & & 95 & 75 & Median & 25 & 5 \\
\hline Sulfate, dissolved (mg/L) & 18 & 2.6 & 1.1 & 1.7 & 2.6 & 2.1 & 1.7 & 1.3 & 1.1 \\
\hline Chloride, dissolved (mg/L) & 18 & 4.5 & .7 & 1.7 & 4.5 & 2.2 & 1.4 & .9 & .7 \\
\hline Dissolved solids, sum of constituents $(\mathrm{mg} / \mathrm{L})$ & 18 & 93 & 32 & 63 & 93 & 79 & 62 & 51 & 32 \\
\hline Nitrate, total as $\mathrm{N}(\mathrm{mg} / \mathrm{L})$ & 17 & .27 & .08 & .15 & .27 & .22 & .11 & .10 & .08 \\
\hline Phosphorus, total (mg/L) & 7 & .14 & .06 & .09 & -- & -- & -- & -- & -- \\
\hline Strontium, dissolved $(\mu \mathrm{g} / \mathrm{L})$ & 18 & 20 & 4.0 & 11 & 20 & 14 & 11 & 8.2 & 4.0 \\
\hline
\end{tabular}

${ }^{1}$ The nth percentile is the value exceeded by no more than $n$ percent of the data.

Table 10. Summary statistics for selected physical properties and water-quality constituents at site 16, Seco Creek at Rowe Ranch near D'Hanis, Texas (08202700), during-and-after implementation period of best-management practices

[Data collected during stormflow conditions. $\mathrm{ft}^{3} / \mathrm{s}$, cubic feet per second; $\mu \mathrm{S} / \mathrm{cm}$, microsiemens per centimeter at 25 degrees Celsius; --, not computed; mg/L, milligrams per liter; BOD, biochemical oxygen demand; cols./100 mL, colonies per 100 milliliters; >, greater than; $\mathrm{CaCO}_{3}$, calcium carbonate; $\mathrm{N}$, nitrogen; $\mu \mathrm{g} / \mathrm{L}$, micrograms per liter]

\begin{tabular}{|c|c|c|c|c|c|c|c|c|c|}
\hline \multirow[b]{2}{*}{ Selected property or constituent } & \multirow{2}{*}{$\begin{array}{c}\text { No. } \\
\text { of } \\
\text { sam- } \\
\text { ples }\end{array}$} & \multirow[b]{2}{*}{$\begin{array}{l}\text { Maxi- } \\
\text { mum }\end{array}$} & \multirow[b]{2}{*}{$\begin{array}{l}\text { Mini- } \\
\text { mum }\end{array}$} & \multirow[b]{2}{*}{ Mean } & \multicolumn{5}{|c|}{ Percentiles $^{1}$} \\
\hline & & & & & 95 & 75 & Median & 25 & 5 \\
\hline Discharge, instantaneous $\left(\mathrm{ft}^{3} / \mathrm{s}\right)$ & 13 & 2,900 & 37 & 618 & 2,900 & 846 & 341 & 146 & 37 \\
\hline Specific conductance $(\mu \mathrm{S} / \mathrm{cm})$ & 12 & 233 & 102 & 157 & 233 & 200 & 144 & 120 & 102 \\
\hline $\mathrm{pH}$ (standard units) & 9 & 8.2 & 7.4 & 7.8 & -- & -- & -- & -- & -- \\
\hline Dissolved oxygen $(\mathrm{mg} / \mathrm{L})$ & 9 & 9.6 & 8.2 & 8.8 & -- & -- & -- & -- & -- \\
\hline BOD, 5-day (mg/L) & 10 & 5.8 & .2 & 3.5 & 5.8 & 4.6 & 3.7 & 2.8 & .2 \\
\hline Coliforms, fecal (cols./100 mL) & 3 & $>160,000$ & 4,400 & -- & -- & -- & -- & -- & -- \\
\hline Streptococci, fecal (cols./100 mL) & 3 & 30,000 & 4,100 & -- & -- & -- & -- & -- & -- \\
\hline Hardness, total as $\mathrm{CaCO}_{3}(\mathrm{mg} / \mathrm{L})$ & 11 & 99 & 46 & 75 & 99 & 93 & 73 & 59 & 46 \\
\hline Calcium, dissolved (mg/L) & 11 & 35 & 17 & 27 & 35 & 33 & 27 & 22 & 17 \\
\hline Magnesium, dissolved (mg/L) & 11 & 3.2 & .8 & 1.9 & 3.2 & 2.6 & 1.6 & 1.1 & .8 \\
\hline Sodium, dissolved (mg/L) & 11 & 4.3 & 1.1 & 2.2 & 4.3 & 3.1 & 1.7 & 1.3 & 1.1 \\
\hline Potassium, dissolved (mg/L) & 11 & 4.1 & 2.0 & 3.0 & 4.1 & 3.7 & 2.8 & 2.2 & 2.0 \\
\hline Sulfate, dissolved (mg/L) & 11 & 12 & 1.7 & 5.8 & 12 & 9.4 & 4.4 & 2.6 & 1.7 \\
\hline Chloride, dissolved (mg/L) & 11 & 7.3 & 1.5 & 3.6 & 7.3 & 5.2 & 3.0 & 2.2 & 1.5 \\
\hline Dissolved solids, sum of constituents (mg/L) & 11 & 124 & 60 & 95 & 124 & 117 & 90 & 77 & 60 \\
\hline Nitrate, total as $\mathrm{N}(\mathrm{mg} / \mathrm{L})$ & 11 & .57 & .06 & .26 & .57 & .37 & .20 & .15 & .06 \\
\hline Phosphorus, total (mg/L) & 11 & .17 & .02 & .11 & .17 & .16 & .13 & .06 & .02 \\
\hline Strontium, dissolved ( $\mu \mathrm{g} / \mathrm{L})$ & 11 & 110 & 21 & 60 & 110 & 95 & 49 & 27 & 21 \\
\hline
\end{tabular}

\footnotetext{
${ }^{1}$ The nth percentile is the value exceeded by no more than $\mathrm{n}$ percent of the data.
} 
Table 11. Summary statistics for selected physical properties and water-quality constituents at site 18, Seco Creek near Yancey, Texas (08202900), during-and-after implementation period of best-management practices

[Data collected during stormflow conditions. $\mathrm{ft}^{3} / \mathrm{s}$, cubic feet per second; $<$, less than; $\mu \mathrm{S} / \mathrm{cm}$, microsiemens per centimeter at 25 degrees Celsius; mg/L, milligrams per liter; BOD, biochemical oxygen demand; --, not computed; cols./100 mL, colonies per 100 milliliters; $\mathrm{CaCO}_{3}$, calcium carbonate; $\mathrm{N}$, nitrogen; $\mu \mathrm{g} / \mathrm{L}$, micrograms per liter]

\begin{tabular}{|c|c|c|c|c|c|c|c|c|c|}
\hline \multirow[b]{2}{*}{ Selected property or constituent } & \multirow{2}{*}{$\begin{array}{l}\text { No. } \\
\text { of } \\
\text { sam- } \\
\text { ples }\end{array}$} & \multirow[b]{2}{*}{$\begin{array}{l}\text { Maxi- } \\
\text { mum }\end{array}$} & \multirow[b]{2}{*}{$\begin{array}{l}\text { Mini- } \\
\text { mum }\end{array}$} & \multirow[b]{2}{*}{ Mean } & \multicolumn{5}{|c|}{ Percentiles $^{1}$} \\
\hline & & & & & 95 & 75 & Median & 25 & 5 \\
\hline Discharge, instantaneous $\left(\mathrm{ft}^{3} / \mathrm{s}\right)$ & 17 & 1,920 & $<0.01$ & 202 & 566 & 62 & 44 & 1.22 & $<0.01$ \\
\hline Specific conductance $(\mu \mathrm{S} / \mathrm{cm})$ & 17 & 1,830 & 106 & 760 & 1,830 & 1,400 & 418 & 303 & 106 \\
\hline pH (standard units) & 15 & 8.3 & 7.0 & 7.8 & 8.3 & 8.0 & 7.8 & 7.6 & 7.0 \\
\hline Dissolved oxygen (mg/L) & 12 & 9.3 & 7.1 & 8.2 & 9.3 & 8.7 & 8.0 & 7.8 & 7.1 \\
\hline BOD, 5-day (mg/L) & 8 & 4.6 & 1.6 & 3.11 & -- & -- & -- & -- & -- \\
\hline Coliforms, fecal (cols./100 mL) & 6 & 250,000 & 570 & 56,000 & -- & -- & -- & -- & -- \\
\hline Streptococci, fecal (cols./100 mL) & 6 & 37,000 & 510 & 14,000 & -- & -- & -- & -- & -- \\
\hline Hardness, total as $\mathrm{CaCO}_{3}(\mathrm{mg} / \mathrm{L})$ & 17 & 480 & 47 & 211 & 480 & 320 & 140 & 96 & 47 \\
\hline Calcium, dissolved (mg/L) & 17 & 140 & 16 & 62 & 140 & 92 & 46 & 32 & 16 \\
\hline Magnesium, dissolved (mg/L & 17 & 38 & 1.8 & 13 & 38 & 25 & 6.2 & 4.1 & 1.8 \\
\hline Sodium, dissolved (mg/L) & 17 & 220 & 2.3 & 72 & 220 & 160 & 28 & 14 & 2.3 \\
\hline Potassium, dissolved (mg/L) & 17 & 8.0 & 2.8 & 5.2 & 8.0 & 6.6 & 5.0 & 3.9 & 2.8 \\
\hline Sulfate, dissolved (mg/L) & 17 & 480 & 7.5 & 150 & 480 & 310 & 57 & 28 & 7.5 \\
\hline Chloride, dissolved (mg/L) & 17 & 230 & 2.8 & 75 & 230 & 120 & 36 & 16 & 2.8 \\
\hline Dissolved solids, sum of constituents (mg/L) & 17 & 1,190 & 77 & 456 & 1,190 & 863 & 248 & 174 & 77 \\
\hline Nitrate, total as N (mg/L) & 16 & 4.97 & .11 & 1.22 & 4.97 & 1.42 & .68 & .49 & .11 \\
\hline Phosphorus, total (mg/L) & 6 & .42 & .06 & .18 & -- & -- & -- & -- & -- \\
\hline Strontium, dissolved $(\mu \mathrm{g} / \mathrm{L})$ & 17 & 1,100 & 60 & 410 & 1,100 & 740 & 210 & 140 & 60 \\
\hline
\end{tabular}

\footnotetext{
${ }^{1}$ The $n$th percentile is the value exceeded by no more than $n$ percent of the data.
} 
Blank Page 


\section{APPENDIX—“Readme" File}




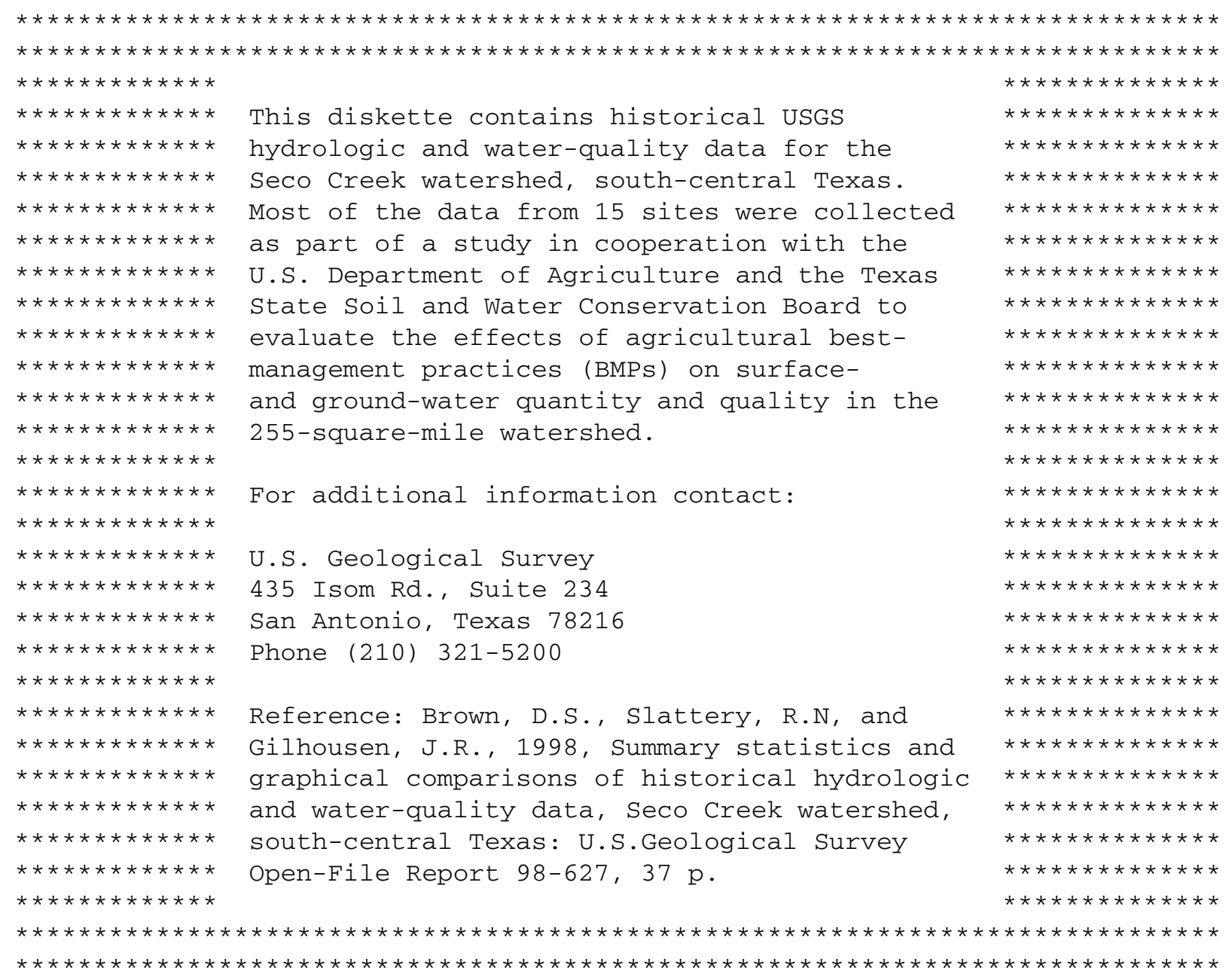

The diskette contains 2 files: readme, data

readme -- This explanation file.

data -- A compressed, self-extracting file of 50 data files. (Instructions for extracting data files: Copy the file "data" from the diskette to a directory on the hard drive and double click on the filename "data" to extract individual files.)

The 50 data files comprise five types of data. Each of the five types of data are in two formats. File names ending with the suffix.rdb are in tabdelimitered ASCII format for use with spreadsheet software. File names ending with the suffix.tbl are in tabular 132-column ASCII format for use with word processing software. The five .rdb and .tbl file types (and the number of files of each type) are

1. sfxxxxx.rdb (5), sfxxxxx.tbl (5) -- Continuous streamflow, daily mean.

2. rcxxxxx.rdb (1), rcxxxxx.tbl (1) -- Continuous reservoir storage, daily maximum. 
3. rfxxxxx.rdb (14), rfxxxxx.tbl (14)-- Continuous rainfall, daily sum.

4. qpbxxxxx.rdb (1), qpbxxxxx.tbl (1) -- Periodic base-flow water quality.

5. qpsxxxxx.rdb (4), qpsxxxxx.tbl (4) -- Periodic stormflow water quality.

CONTENTS OF THE REMAINDER OF THIS README FILE:

1. LIST OF USGS SITES AND DATA TYPE AVAILABLE

2. FORMATS AND CONTENTS FOR CONTINUOUS STREAMFLOW, DAILY MEAN FILES

Tab-delimitered files (sfxxxxx.rdb)

Table files (sfxxxxx.tbl)

Daily Mean Values

Statistics of Monthly Mean Data

Summary statistics

Explanation of Terms

3. FORMATS FOR CONTINUOUS RESERVOIR STORAGE, DAILY MAXIMUM FILES

Tab-delimitered file (rcxxxxx.rab)

Table file (rcxxxxx.tbl)

4. FORMATS FOR CONTINUOUS RAINFALL, DAILY SUM FILES

Tab-delimitered files (rfxxxxx.rdb)

Table files (rfxxxxx.tbl)

5. FORMATS FOR WATER-QUALITY DATA FILES

Tab-delimitered files (qpbxxxxx.rdb and qpsxxxxx.rdb)

Table files (qpbxxxxx.tbl and qpsxxxxx.tbl)

Explanation of Hydrologic Condition Codes, Remark Codes, Units

of Measure, and STORET codes

1. LIST OF USGS SITES AND DATA TYPE AVAILABLE

The table below is a list of sites, the type of data for each site, and the period of record of data for each site. The numbers in the first column correspond to site numbers on figure 2 in the report. The last five digits of the station number correspond to the five digits of the file name. The period of record is in water years.

[rf, rainfall; sf, streamflow; qpb, periodic base-flow water quality; qps, periodic stormflow water quality; rc, reservoir content]

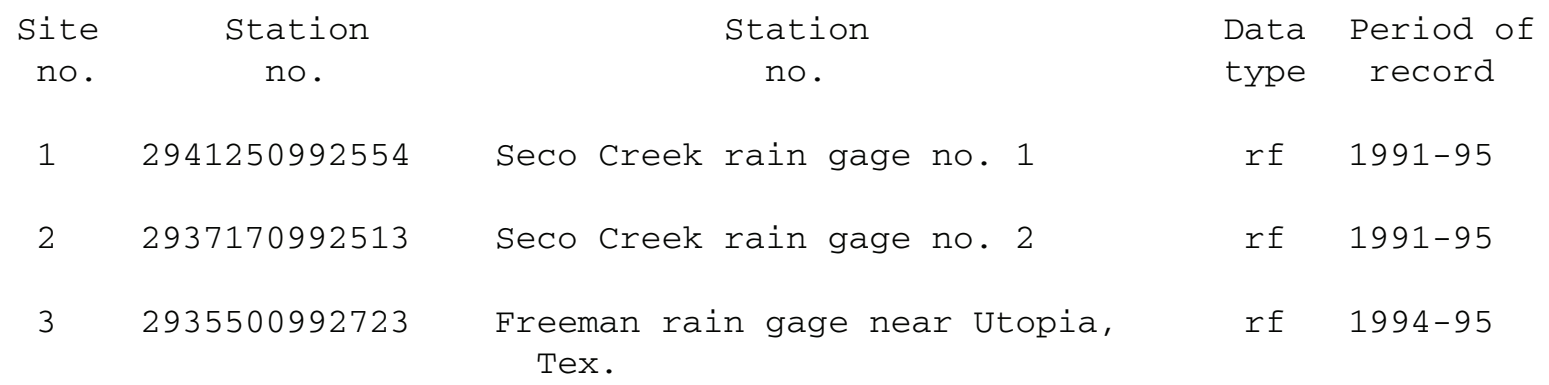




\begin{tabular}{|c|c|c|c|c|}
\hline 4 & 08201500 & $\begin{array}{l}\text { Seco Creek at Miller Ranch near } \\
\text { Utopia, Tex. }\end{array}$ & $\begin{array}{l}\text { rf } \\
\text { sf } \\
\text { qpb }\end{array}$ & $\begin{array}{l}1988-95 \\
1961-95 \\
1970-95\end{array}$ \\
\hline 5 & 2933550992019 & $\begin{array}{l}\text { Coffee rain gage near Utopia, } \\
\text { Tex. }\end{array}$ & $r f$ & $1994-95$ \\
\hline 6 & 08202450 & $\begin{array}{l}\text { Seco Creek Reservoir Inflow } \\
\text { near Utopia, Tex. }\end{array}$ & $\begin{array}{l}\text { rf } \\
\text { sf } \\
\text { qps }\end{array}$ & $\begin{array}{l}1991-95 \\
1991-95 \\
1991-95\end{array}$ \\
\hline 9 & 2931150991651 & $\begin{array}{l}\text { Brown rain gage near D'Hanis, } \\
\text { Tex. }\end{array}$ & $r f$ & 1995 \\
\hline 10 & 2928080992030 & $\begin{array}{l}\text { Valdina Farms rain gage near } \\
\text { D'Hanis, Tex. }\end{array}$ & $r f$ & $1994-95$ \\
\hline 11 & 2928480991442 & Parkers Creek rain gage no. 1 & $r f$ & $1991-95$ \\
\hline 12 & 08202790 & $\begin{array}{l}\text { Parkers Creek Reservoir Inflow } \\
\text { near D'Hanis, Tex. }\end{array}$ & $\begin{array}{l}\text { rf } \\
\text { sf } \\
\text { gps }\end{array}$ & $\begin{array}{l}1991-95 \\
1991-95 \\
1991-95\end{array}$ \\
\hline 13 & 08202800 & $\begin{array}{l}\text { Parkers Creek Reservoir near } \\
\text { D'Hanis, Tex. }\end{array}$ & rc & $1991-95$ \\
\hline 15 & 2925270991837 & $\begin{array}{l}\text { Chaney rain gage near D'Hanis, } \\
\text { Tex. }\end{array}$ & $r f$ & $1994-95$ \\
\hline 16 & 08202700 & $\begin{array}{l}\text { Seco Creek at Rowe Ranch near } \\
\text { D'Hanis, Tex. }\end{array}$ & $\begin{array}{l}\text { rf } \\
\text { sf } \\
\text { gps }\end{array}$ & $\begin{array}{l}1994-95 \\
1961-95 \\
1991-93\end{array}$ \\
\hline 17 & 2914540991642 & $\begin{array}{l}\text { Arnold rain gage near D'Hanis, } \\
\text { Tex. }\end{array}$ & $r f$ & $1994-95$ \\
\hline 18 & 08202900 & Seco Creek near Yancey, Tex. & $\begin{array}{l}\text { rf } \\
\text { sf } \\
\text { qps }\end{array}$ & $\begin{array}{l}1992-95 \\
1991-95 \\
1991-95\end{array}$ \\
\hline
\end{tabular}

2. FORMATS AND CONTENTS FOR CONTINUOUS STREAMFLOW, DAILY MEAN FILES sfxxxxx.rdb files -- The first three tab-delimetered columns are the date with the year, month, and day separated by tabs. Dates are in ascending order for the period of available record. The fourth column is the daily-mean discharge for that date in cubic feet per second. Missing record is indicated by a negative value.

sfxxxxx.tbl files -- The 132-column table file has three parts: (1) Daily mean values; (2) Statistics of monthly mean data; and (3) Summary statistics. Each part is described below: 
Daily Mean Values

The daily mean values table for streamflow-gaging stations lists mean discharge for each day in cubic feet per second. Estimated daily mean values are indicated by "e" preceding the value. Missing record is indicated by ---. In the monthly summary below the daily mean table, the TOTAL line lists the sum of the daily values. The MEAN line lists the average flow in cubic feet per second during the month. MAX, MIN, and MED list the maximum, minimum, and median daily discharges, respectively, for the month. Discharge for the month also may be expressed in acre-feet (on the drainage area upstream of the gaging station) (AC-FT), or in cubic feet per second per square mile (of drainage area upstream of the gaging station) (CFSM), or in inches (on the drainage area upstream of the gaging station) (IN.).

Statistics of Monthly Mean Data

A tabular summary of the mean (MEAN), maximum (MAX), and minimum (MIN) monthly mean discharges for each month for a designated period of water years is listed with the water years of the first occurrence of the maximum and minimum monthly mean discharges immediately below.

\section{Summary statistics}

The SUMMARY STATISTICS table is a statistical summary of annual, daily, and instantaneous discharges for the previous calendar year, designated water year, and period of record through the designated water year.

Annual period-of-record statistics are computed from data for complete-record water years, except ANNUAL SEVEN-DAY MINIMUM, which includes data for partialrecord water years (if any). The other period-of-record statistics also include data for partial-record water years (if any).

The date or water year, as appropriate, of the first occurrence of each statistic of an extreme value of discharge is provided adjacent to the statistic. Runoff data also are listed unless the drainage basin has extensive regulation or diversion of flow.

Explanation of Terms

ANNUAL TOTAL

The sum of the daily mean discharges for the year.

ANNUAL MEAN

The arithmetic mean of individual daily mean discharges for the year noted or for the designated period.

HIGHEST ANNUAL MEAN

The maximum annual mean discharge for the designated period.

LOWEST ANNUAL MEAN

The minimum annual mean discharge for the designated period. 
HIGHEST DAILY MEAN

The maximum daily mean discharge for the year or for the designated period.

LOWEST DAILY MEAN

The minimum daily mean discharge for the year or for the designated period.

ANNUAL SEVEN-DAY MINIMUM

The lowest mean discharge for 7 consecutive days for a calendar year or a water year. Most low-flow frequency analyses of annual 7-day minimum flows use a climatic year (April 1-March 31). The date shown in the summary statistics table is the initial date of the 7-day period.

INSTANTANEOUS PEAK FLOW

The maximum instantaneous discharge for the water year or for the designated period.

INSTANTANEOUS PEAK STAGE

The maximum instantaneous stage for the water year or for the designated period.

ANNUAL RUNOFF (AC-FT), (CFSM), (INCHES)

Indicates the total quantity of runoff for the drainage area upstream of the gage for the year.

\section{PERCENT EXCEEDS}

The discharge that has been exceeded 10 percent of the time for the designated period.

50 PERCENT EXCEEDS

The discharge that has been exceeded 50 percent of the time for the designated period.

\section{PERCENT EXCEEDS}

The discharge that has been exceeded 90 percent of the time for the designated period.

WATER YEAR (WY)

The 12-month period October 1 through September 30. The water year is designated by the calendar year in which it ends. Thus, the year ending September 30, 1995, is water year 1995.

\section{FORMATS FOR CONTINUOUS RESERVOIR STORAGE, DAILY MAXIMUM FILES}

rcxxxxx.rdb file -- The first three tab-delimetered columns are the date with the year, month, and day separated by tabs. Dates are in ascending order for the period of available record. The fourth column is the daily maximum for that date, in acre-feet. Missing record is indicated by a negative value.

rcxxxxx.tbl file -- The 132-column table file comprises daily maximum values, in acre-feet for the given water year, followed by monthly totals and maximums, and calendar year and water year totals and monthly maximums. 
Estimated daily maximum values are indicated by "e" preceding the value. Missing record is indicated by ---.

\section{FORMATS FOR CONTINUOUS RAINFALL, DAILY SUM FILES}

rfxxxxx.rdb files -- The first three tab-delimetered columns are the date with the year, month, and day separated by tabs. Dates are in ascending order for the period of available record. The fourth column is the daily total for that date, in inches. Missing record is indicated by a negative value.

rfxxxxx.tbl files -- The 132-column table file comprises daily sum values, in inches for the given water year, followed by monthly totals and water year totals. Estimated daily sum values are indicated by "e" preceding the value. Missing record is indicated by ---.

\section{FORMATS FOR WATER-QUALITY DATA FILES}

qpbxxxxx.rdb and qpsxxxxx.rdb files -- These tab-delimetered files contain the following data columns: (Each row is a single sample set.)

STAID - Station identification number

DATES - Date sample was collected (format YYYYMMDD)

TIMES - Time (24-hour) sample was collected (format HHMM)

HSTAT - Hydrologic condition code (see list at end of section)

STORET CODE (U.S. Environmental Protection Agency 5-digit identifier for property or constituent) - Each code heads a column for 1 of 165 properties or constituents. STORET codes with descriptions are listed at the end of this section. Values of properties or constituents might be accompanied by a remark code (see list at end of section).

The station identification number, time, and STORET code are shown without leading zeros.

qpbxxxxx.tbl and qpsxxxxx.tbl files -- 132-column tables. The table header lists the station number, station name, and period of record available in the file. The tables list the date and time of samples, hydrologic condition and discharge when samples were obtained, and as many as 165 water-quality properties or constituents and STORET codes. Values of properties and constituents might be accompanied by a remark code.

Explanation of Hydrologic Condition Codes, Remark Codes, Units of Measure, and STORET Codes

Hydrologic condition codes:
4 - Stable, low stage
8 - Rising stage
9 - Stable, normal stage
7 - Peak stage
6 - Stable, high stage
5 - Falling stage 


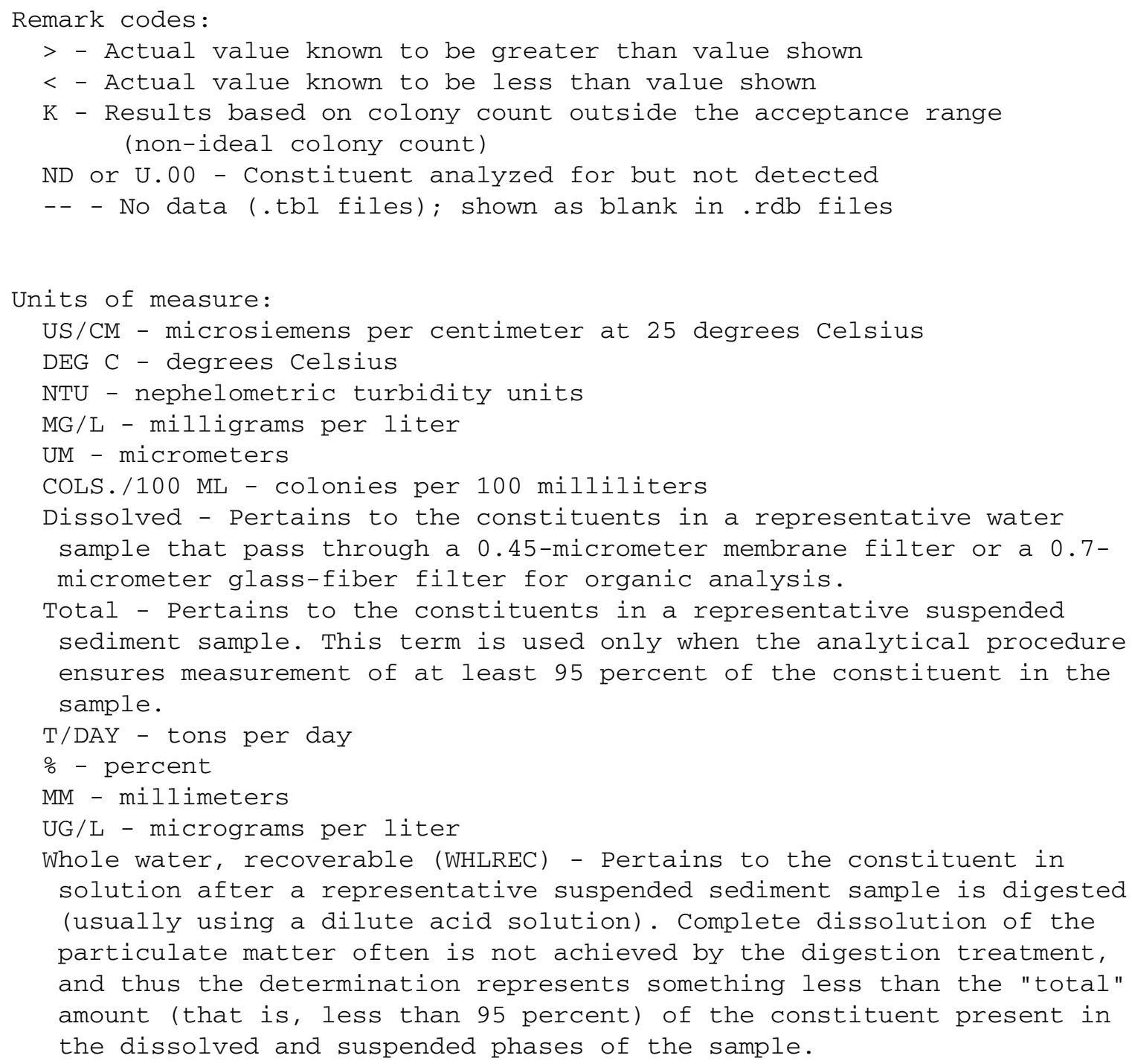




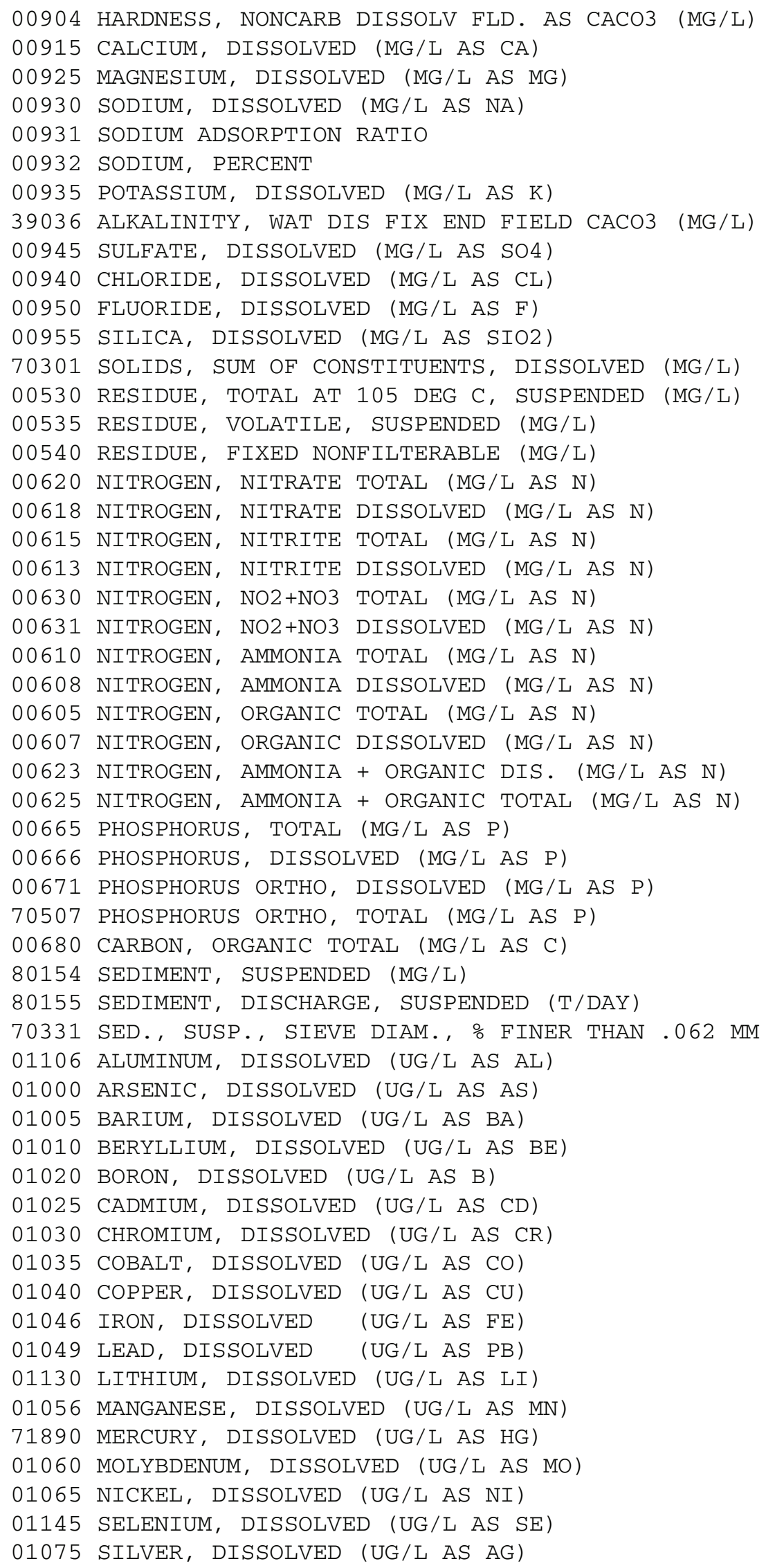


01080 STRONTIUM, DISSOLVED (UG/L AS SR)

01085 VANADIUM, DISSOLVED (UG/L AS V)

01090 ZINC, DISSOLVED (UG/L AS ZN)

30234 BROMACIL, WATER WHLREC (UG/L)

30235 BUTACHLOR, WATER WHLREC (UG/L)

30236 BUTYLATE, WATER WHLREC (UG/L)

30245 CARBOXIN, WATER WHOLE RECOVERABLE (UG/L)

30254 CYCLOATE, WATER WHOLE RECOVERABLE (UG/L)

30255 DIPHENAMID, WATER WHOLE RECOVERABLE (UG/L)

30264 HEXAZINONE, WATER WHOLE RECOVERABLE (UG/L)

30295 PROPACHLOR, WATER WHOLE RECOV. (UG/L)

30311 TERBACIL, WATER WHOLE RECOV. (UG/L)

30324 VERNOLATE, WATER WHOLE RECOV. (UG/L)

34030 BENZENE, TOTAL (UG/L)

32104 BROMOFORM, TOTAL (UG/L)

32102 CARBON TETRACHLORIDE, TOTAL (UG/L)

34301 CHLOROBENZENE, TOTAL (UG/L)

32105 CHLORODIBROMOMETHANE, TOTAL (UG/L)

34311 CHLOROETHANE, TOTAL (UG/L)

32106 CHLOROFORM, TOTAL (UG/L)

34418 METHYLCHLORIDE, TOTAL (UG/L)

34704 CIS-1,3-DICHLOROPROPENE, TOTAL (UG/L)

32101 DICHLOROBROMOMETHANE, TOTAL (UG/L)

34668 DICHLORODIFLUOROMETHANE, TOTAL (UG/L)

34371 ETHYLBENZENE, TOTAL (UG/L)

34413 METHYL BROMIDE, TOTAL (UG/L)

34423 METHYLENE CHLORIDE, TOTAL (UG/L)

77128 STYRENE, TOTAL (UG/L)

34475 TETRACHLOROETHYLENE, TOTAL (UG/L)

34010 TOLUENE, TOTAL (UG/L)

34699 TRANS-1, 3-DICHLOROPROPENE, TOTAL (UG/L)

39030 TRIFLURALIN, TOTAL RECOVER (UG/L)

39180 TRICHLOROETHYLENE, TOTAL (UG/L)

34488 TRICHLOROFLUOROMETHANE, TOTAL (UG/L)

39175 VINYL CHLORIDE, TOTAL (UG/L)

34501 1,1-DICHLOROETHYLENE, TOTAL (UG/L)

34496 1,1-DICHLOROETHANE, TOTAL (UG/L)

34506 1,1,1-TRICHLOROETHANE, TOTAL (UG/L)

34511 1, 1,2-TRICHLOROETHANE, TOTAL (UG/L)

34516 ETHANE, 1, 1,2,2-TETRACHLORO, WAT UNF REC (UG/L)

34536 1,2-DICHLOROBENZENE, WATER UNFLTRD REC (UG/L)

32103 1,2-DICHLOROETHANE, TOTAL (UG/L)

34541 1,2-DICHLOROPROPANE, TOTAL (UG/L)

34566 BENZENE, 1,3-DICHLORO, WATER UNFLTRD REC (UG/L)

34561 1,3-DICHLOROPROPENE, TOTAL (UG/L)

34571 BENZENE, 1,4-DICHLORO, WATER UNFLTRD REC (UG/L)

34546 1,2-TRANSDICHLOROETHENE, TOTAL (UG/L)

34576 2-CHLOROETHYL VINYL ETHER, TOTAL (UG/L)

81551 XYLENE, WATER UNFLTRD REC (UG/L)

39516 PCB, TOTAL (UG/L)

39250 PCNS, UNFILT RECOVER (UG/L)

39330 ALDRIN, TOTAL (UG/L)

82184 AMETRYNE, TOTAL (UG/L)

39630 ATRAZINE, WATER UNFLTRD REC (UG/L) 


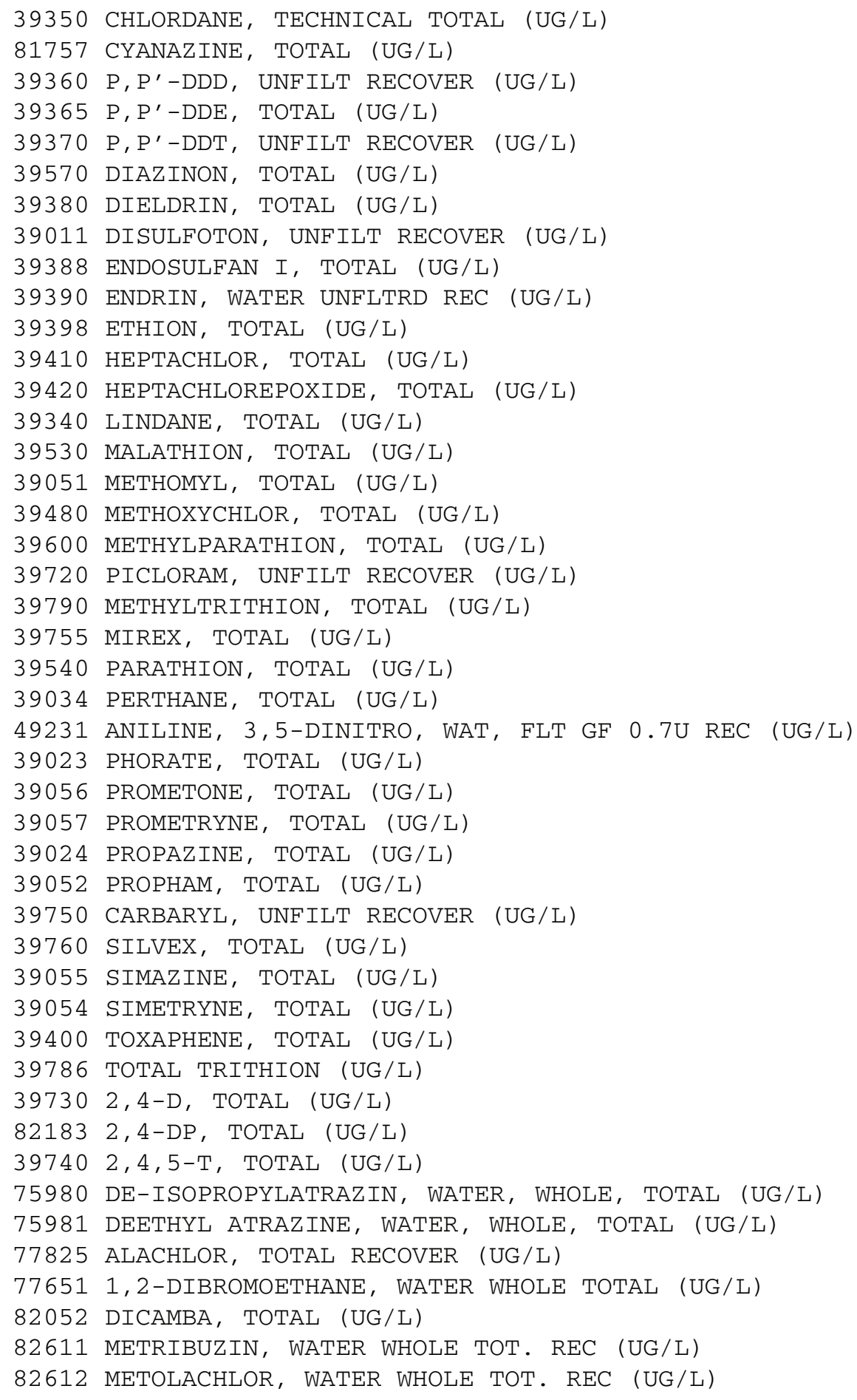

